\title{
Allocation with Demand Competition: Uniform, Proportional, and Lexicographic Mechanisms
}

\author{
Jianbin Li, ${ }^{1}$ Niu Yu, ${ }^{2}$ Zhixin Liu ${ }^{(1)},{ }^{3}$ Xueyuan Cai ${ }^{1}$ \\ ${ }^{1}$ Department of Operations Management, School of Management, Huazhong University of Science and Technology, \\ Wuhan, Hubei 430074, China \\ ${ }^{2}$ Department of Logistics Management, School of Management, Wuhan Textile University, Wuhan, Hubei 430073, China \\ ${ }^{3}$ Department of Management Studies, College of Business, University of Michigan-Dearborn, Dearborn, \\ Michigan 48126-2638, United States
}

Received 2 December 2014; revised 16 March 2017; accepted 16 March 2017

DOI 10.1002/nav.21734

Published online 21 April 2017 in Wiley Online Library (wileyonlinelibrary.com).

\begin{abstract}
We examine capacity allocation mechanisms in a supply chain comprising a monopolistic supplier and two competing retailers with asymmetric market powers. The supplier allocates limited capacity to retailers according to uniform, proportional, or lexicographic mechanism. We study the impact of these allocation mechanisms on supplier pricing decisions and retailer ordering behavior. With individual order size no greater than supplier capacity, we show that all three mechanisms guarantee equilibrium ordering. We provide precise structures of retailer ordering decisions in Nash and dominant equilibria. Further, we compare the mechanisms from the perspective of the supplier, the retailers, and the supply chain. We show that regardless of whether retailer market powers are symmetric, lexicographic allocation with any priority sequence of retailers is better than the other two mechanisms for the supplier. Further, under lexicographic allocation, the supplier gains more profit by granting higher priority to the retailer with greater market power. We also extend our study to the case with multiple retailers. (c) 2017 Wiley Periodicals, Inc. Naval Research Logistics 64: 85-107, 2017
\end{abstract}

Keywords: capacity allocation; uniform allocation; proportional allocation; lexicographic allocation; supply chain; Nash equilibrium

\section{INTRODUCTION}

Capacity shortfall frequently occurs in various industries when retailers' total order size exceeds a supplier's available capacity. For example, capacity shortages often arise in the fashion goods, telecommunications, and electricity industries [8]. Also, it is common practice for an automobile manufacturer to sell through multiple dealers in the same geographic region, who compete for both the manufacturer's limited supply capacity and customer demand for popular vehicle models [10]. However, capacity expansion is often costly and difficult to achieve, particularly for such products as vehicles and seasonal products. Thus, the supplier must price and allocate scarce capacity effectively. In this work, we study how various allocation mechanisms affect supplier pricing and retailer ordering decisions, and how the supplier chooses

Correspondence to: Niu Yu (nyu@wtu.edu.cn) an allocation mechanism with pricing decisions to increase profit.

Specifically, we investigate a two-echelon supply chain in which a monopolistic supplier (he) sells through two competing retailers (she) with demand competition. The capacity allocation mechanisms considered work in the following way. First, the supplier announces his capacity level, the unit wholesale price of this capacity, an allocation rule that defines how capacity will be allocated as a function of retailer order sizes, and a requirement that no order can be more than total capacity. Second, the retailers place their orders. Third, the supplier allocates capacity to retailers using the preannounced allocation rule. Finally, the retailers sell the received capacity to their customers. In our framework, an allocation rule applies within an allocation mechanism. We henceforth refer to a mechanism with a specific rule (e.g., uniform) by its specific rule name (in this case, uniform allocation mechanism). 
The problem under investigation fits within two strategic interactions in a multistage noncooperative game. The vertical interaction is modeled as a Stackelberg game between the supplier as the leader and the two retailers as followers. We assume that the supplier's capacity is given exogenously, and the supplier's decisions are limited to the allocation rule and wholesale price. The horizontal interaction captures the competition between the retailers, who are in the same market and compete for both the supplier's capacity and demand from a common customer population. We consider Cournot competition, in which the two retailers sell at retail prices as functions of the total capacity provided in the retail market by the two retailers. The two retailers have asymmetric market powers, where the retailer with greater market power, high-type retailer, sells at a higher price than the other lowtype retailer. The difference between the market powers of the two retailers is referred to as competitive gap.

Three widely studied allocation rules are considered: uniform, proportional, and lexicographic. Under uniform allocation, if a retailer orders no more than the equal share of total available capacity, then she receives her order and other retailers share the remaining capacity in a similar way. Uniform allocation is individually unresponsive (IU), in that allocation is not strictly increasing with order size. When retailers are local monopolists, uniform allocation guarantees equilibrium orders and is truth-inducing in that it incentivizes retailers to order their individually optimal order sizes, thus eliminating the gaming effect $[1,2,11]$. However, when retailers compete for customer demand, even though uniform allocation still warrants equilibrium orders, it does not prevent the gaming effect $[6,10]$.

Proportional allocation is more intuitive in that it allocates capacity in proportion to order size; it thus strictly increases with order size and is individually responsive (IR). Even for monopolistic retailers, proportional allocation does not guarantee equilibrium orders and is well-known for causing order inflation $[1,2,9]$.

Under lexicographic allocation, retailers are prioritized such that the retailer with the highest priority always has her order filled first. Lexicographic allocation is a type of IU allocation. Cachon and Lariviere [1] demonstrate that lexicographic allocation is truth-inducing and guarantees equilibrium orders when retailers face independent demand. When retailers compete for demand, equilibrium orders still exist under lexicographic allocation, but the gaming effect may also occur [4]. Hence, lexicographic allocation behaves similarly to uniform allocation with respect to the existence of equilibrium orders and the gaming effect.

Another popular IR allocation rule is linear allocation, which allocates each retailer her order size minus a common deduction. Linear allocation performs similarly to proportional allocation in the presence of equilibrium orders and the gaming effect $[1,10]$. In fact, consistent with the findings of [10] in a similar problem setting, linear allocation performs nearly identically to proportional allocation for our problem; hence, we omit this allocation.

The prespecified upper bound on order size, total capacity, is practically meaningful, as the supplier's capacity is publicly known, and ordering more than supplier capacity obviously exceeds the supplier's capability to fill the order [4]. Further, allowing unbounded orders can induce arbitrarily large orders from competing retailers, leading to unpredictable allocations $[1,2]$. The upper bound can also be considered as embedded in allocation rules. That is, any order larger than the upper bound will be truncated to the bound.

Capacity allocation is studied extensively in the operations literature. Hall and Liu [7] survey the problem and provide classification schemes based on whether a game is cooperative or noncooperative, capacity is sufficient or insufficient, single or multiple types of capacity are considered, the timing issue is addressed, and what kinds of mechanisms (including auctions, contracts, pricing, and rules) are used. Our problem is a type of noncooperative game with a single type of deficient capacity allocated through rules without timing issues. We next review the literature on this problem class.

Sprumont [11] establishes important theories for rulebased allocations. This author shows that for a general capacity allocation problem where each retailer has a single-peaked preference for capacity size, uniform allocation is the unique rule that is truth-inducing, efficient, and anonymous; here, efficiency means that if total order size is greater (less) than the available capacity, then no retailer receives an allocation greater (less) than her order, and anonymity means that retailers with equal orders receive the same allocation. Lee et al. [9] show that, despite its intuitive attraction and popularity, proportional allocation can lead to order inflation and contributes to the well-known bullwhip effect. Cachon and Lariviere $[1,2]$ analyze equilibrium ordering decisions, supplier capacity choice, and supply chain performance under various allocation mechanisms, including linear, proportional, lexicographic, and uniform. These authors find that, with asymmetric information, proportional allocation is not resistant to the gaming effect and may distort supplier capacity selection. Uniform and lexicographic allocations are both truth-inducing, but may result in lower profit for supply chain members. Cachon and Lariviere [3] investigate a two-period allocation using a turn and earn mechanism, where allocation in the current period is based on sales in the preceding period. These authors show that this mechanism can increase the supplier's profit at the cost of the retailers, and even the supply chain. Chen et al. [5] study the gaming effect caused by proportional allocation, using laboratory experiments. These authors find that retailers often order much less than the Nash equilibrium. They propose a bounded rationality model and find that retailers learn to be more rational through repeated games, but their orders still may not converge to Nash equilibrium. 
A common assumption in the aforementioned literature is that each retailer is a local monopolist and her demand is independent from other retailers. To our knowledge, only four papers consider capacity allocation with demand competition. Liu [10] considers demand competition between two competing retailers where retail prices are linear functions of sales volume. This author investigates how different allocations affect supplier, retailers, and supply chain profits. Liu finds that a supplier can sell more with less capacity, and retailers may earn more when the supplier has less capacity. Cho and Tang [6] extend Liu [10] to a case with multiple retailers, focusing on the gaming effect caused by uniform allocation. These authors identify exact conditions under which the gaming effect is present. They then propose a competitive allocation that eliminates the gaming effect under demand competition. Chen et al. [4] analyze supplier wholesale pricing decisions under proportional and lexicographic allocations, with retailers possessing identical market power in demand competition. These authors show that lexicographic allocation can bring higher profit to both the supplier and the supply chain. Yang et al. [12] consider a supplier who sells both by himself and through a retailer, and thus allocates capacity between himself and the retailer. These authors find that the supplier, the retailer, and consumers may simultaneously benefit from the supplier's limited capacity.

We now clarify our contribution relative to $[2,4,6,10]$. First, Cachon and Lariviere [2] assume local monopolistic retailers, while we consider the case where retailers engage in demand competition such that a retailer's profit depends on allocations to all retailers. Besides, we endogenize the supplier's wholesale price as his decision variable. Second, Cho and Tang [6] and Liu [10] consider demand competition among retailers, but do not consider lexicographic allocation or wholesale pricing decisions, as we do. Third, Chen et al. [4] consider identical retailers, while our consideration of asymmetric retailers is more general, and we study uniform allocation that is not considered in [4].

Overall, using a more general model setting, we regard our contribution as unifying and generalizing various results from the literature on capacity allocation with demand competition, as follows:

- We endogenize the supplier's wholesale price as a decision variable and consider all three common allocation mechanisms: uniform, proportional, and lexicographic.

- We consider demand competition among retailers with asymmetric market powers.

- We consider cases with two and multiple retailers, and analytically characterize the relative performance of the three mechanisms for the supplier, the retailers, and the supply chain.
Specifically, we show that under uniform or proportional mechanism, when the wholesale price is relatively low, each retailer places an order equal to the prespecified upper bound, and thus receives half the capacity. However, lexicographic mechanism performs differently; although both retailers prefer greater allocation, the retailer with higher priority can receive the entire capacity. Conversely, if the wholesale price is sufficiently high, then under all mechanisms considered, the retailer with lower market power would be driven out of the market. For the supplier, we find that the wholesale pricing decision is sensitive to his capacity level and the competitive gap. Among the three mechanisms, lexicographic allocation is best for the supplier, and proportional allocation outperforms uniform allocation. Specifically, for any given wholesale price, the supplier can sell at least the same size of capacity from lexicographic allocation as from the other two allocations. Further, using lexicographic allocation, the supplier can sell more capacity by granting higher priority to the high-type retailer, especially when the competitive gap is large. We also extend our study to the case with multiple retailers. We analytically show that proportional allocation dominates uniform allocation and lexicographic allocation with a priority sequence of retailers in nonincreasing market powers dominates proportional allocation from the perspective of the supplier for any number of retailers. We numerically show that lexicographic allocation with any priority sequence of retailers dominates uniform allocation for any number of retailers. With multiple retailers, for the supplier, the relative performance of lexicographic allocation compared with proportional allocation depends on the priority sequence of retailers: lexicographic allocation may outperform (underperform) proportional allocation if priority is given to retailers with higher (lower) market power. We also analytically characterize the relative performance of the three allocation mechanisms for both the retailers and the supply chain.

This article proceeds as follows. In Section 2, we formally describe our model, specifically with two retailers. Sections 3 and 4 conduct equilibrium analysis for retailer ordering and supplier wholesale pricing decisions under uniform, proportional, and lexicographic mechanisms, respectively. In Section 5, we compare the three mechanisms from the perspective of the supplier, the retailers and the supply chain. Results are developed for extended cases with multiple retailers in Section 6. Section 7 summarizes our work. All proofs are in the Appendix.

\section{THE MODEL}

Consider a two-echelon supply chain consisting of a single supplier and two downstream retailers. The retailers order a single type of product from the supplier, who has a fixed 
capacity in the quantity of $K$. Each retailer will receive her ordered quantity if capacity is ample. However, when the supplier's capacity is limited such that it cannot satisfy the sum of orders, an allocation rule should be implemented to allocate the capacity between the two retailers. Suppose retailer $i$, who places an order with quantity $r_{i}(i=1,2)$ receives an allocation with quantity $q_{i}\left(r_{1}, r_{2}\right)$. Without loss of generality, the supplier's production cost associated with unit capacity is normalized to zero for succinctness. The supplier charges a wholesale $w$ for each unit of capacity sold. As discussed in Section 1 and as discussed in [4], we do not allow a retailer's order size to exceed total capacity, that is, we require $r_{i} \leq K$. After receiving allocations, the retailers in turn sell the product to the same market and compete for customer demand. Following widely adopted convention in the capacity allocation literature, we assume that information is symmetric and complete for all parties, that is, supplier capacity, unit capacity wholesale price, the allocation rule, the upper bound for each individual order, and the customer demand functions are common knowledge of the supplier and retailers.

Let $p_{i}$ be the retail price of retailer $i(i=1,2)$. The retail price is determined by a linear demand function

$$
p_{i}=z_{i}-q_{1}\left(r_{1}, r_{2}\right)-q_{2}\left(r_{1}, r_{2}\right),
$$

where $z_{i}$ represents retailer $i$ 's market power, which may capture retailer $i$ 's comparative advantage over the other retailer because of certain factors, such as ease of access, customer preference, and brand equity. Without loss of generality, we assume $z_{1} \geq z_{2}$, and refer to retailer 1 as a high-type retailer and retailer 2 as a low-type retailer.

Note that we characterize a duopoly model of two retailers, and it is necessary to have $z_{2}>\left(z_{1}+w\right) / 2$ to retain both retailers in the market when the supplier has ample capacity; otherwise, retailer 2 cannot survive in competition with retailer 1 . Such an assumption is adopted by $[6,10]$. However, in our problem $w$ is a decision variable of the supplier, and it would be artificial to impose a lower bound on $w$ other than 0 . Henceforth, we assume $z_{2}>z_{1} / 2$.

We next describe the sequence of events and decisions. First, before the selling season starts, the supplier announces his capacity level, unit wholesale price of the capacity, an allocation rule, and an upper bound equal to his capacity size for any order. Second, the retailers place their orders. Third, the supplier allocates capacity to retailers using the preannounced allocation rule. Finally, the retailers sell the allocated capacity to their customers. This is a Stackelberg game in which the supplier is the leader and the retailers are followers. For a given capacity level and allocation rule, the supplier's problem is to choose a wholesale price $w$ to maximize his profit:

$$
\Pi_{s}=\max _{w}\left\{w \cdot\left(q_{1}\left(r_{1}, r_{2}\right)+q_{2}\left(r_{1}, r_{2}\right)\right)\right\} .
$$

Naval Research Logistics DOI 10.1002/nav
Given the wholesale price, capacity level, and allocation rule, the retailers' problems are

$$
\begin{aligned}
\Pi_{i}\left(r_{j}, w\right)= & \max _{r_{i}}\left\{\left(z_{i}-q_{i}\left(r_{i}, r_{j}\right)-q_{j}\left(r_{i}, r_{j}\right)\right.\right. \\
& \left.-w) \cdot q_{i}\left(r_{i}, r_{j}\right)\right\},
\end{aligned}
$$

where $i, j=1,2$ and $i \neq j$.

Here, we consider three commonly used allocation rules: uniform, proportional, and lexicographic allocations. First, consider two retailers under uniform allocation. A retailer receives her order if she orders less than half of the capacity, and the other retailer receives the minimum of her order and the remaining capacity; if the retailer with smaller order size orders more than half the capacity, then she will share the capacity equally with the other retailer. Formally, uniform allocation with two retailers is defined as follows:

$q_{i}\left(r_{i}, r_{j}\right)= \begin{cases}r_{i} & \text { if } r_{i}+r_{j} \leq K, \\ \min \left\{r_{i}, K / 2\right\} & \text { if } r_{i}+r_{j}>K, r_{i} \leq r_{j}, \\ \max \left\{K-r_{j}, K / 2\right\} & \text { if } r_{i}+r_{j}>K, r_{i}>r_{j},\end{cases}$

where $i, j=1,2$ and $i \neq j$.

Under proportional allocation, a retailer receives the allocation size she orders if the capacity is sufficient; otherwise, she obtains allocation in proportion to her order size. Hence,

$$
q_{i}\left(r_{i}, r_{j}\right)= \begin{cases}r_{i} & \text { if } r_{i}+r_{j} \leq K, \\ \frac{K r_{i}}{r_{i}+r_{j}} & \text { if } r_{i}+r_{j}>K,\end{cases}
$$

where $i, j=1,2$ and $i \neq j$.

Under lexicographic allocation, the supplier grants priority to one of the retailers and satisfies her order first as far as possible; the supplier then allocates the remaining capacity to the other retailer. Suppose priority is given to retailer $i$ and $r_{i}, r_{j} \leq K$. We have

$$
\begin{aligned}
& q_{i}\left(r_{i}, r_{j}\right)=r_{i} \text { and } \\
& q_{j}\left(r_{i}, r_{j}\right)= \begin{cases}r_{j} & \text { if } r_{i}+r_{j} \leq K, \\
K-r_{i} & \text { if } r_{i}+r_{j}>K,\end{cases}
\end{aligned}
$$

where $i, j=1,2$ and $i \neq j$.

Before examining the allocation mechanisms, we first derive order/allocation sizes in equilibrium when the supply of product is unlimited. In this case, retailer $i$ 's order quantity equals her capacity allocation size. We do not allow capacity to be withheld by retailers, and the allocation size is the same as the selling quantity to the market. Such an assumption is used by $[2,4,6,10]$. From retailer $i$ 's profit function $\pi_{i}\left(r_{1}, r_{2}\right)=\left(z_{i}-w-r_{1}-r_{2}\right) r_{i}, i=1,2$, we obtain the equilibrium order quantity without capacity constraint:

$$
r_{1}^{*}=\frac{2 z_{1}-z_{2}-w}{3} \text {, and } r_{2}^{*}=\frac{2 z_{2}-z_{1}-w}{3} .
$$


In addition, $q_{i}^{*}=r_{i}^{*}$, and the total allocated capacity is $q_{1}^{*}+q_{2}^{*}=\frac{z_{1}+z_{2}-2 w}{3}$.

For convenience, in our analysis, we adopt the following notations. First, we map real value interval to nonnegative interval as

$$
[a, b]= \begin{cases}\emptyset & \text { if } b<a \text { or } b<0 ; \\ {[0, b]} & \text { if } b>0 \text { and } a<0 \\ {[a, b]} & \text { if } 0 \leq a \leq b .\end{cases}
$$

Also, we denote $\left[x_{1}, x_{2}\right] \times\left[y_{1}, y_{2}\right]$ orders $(a, b)$ for any $x_{1} \leq a \leq x_{2}$ and $y_{1} \leq b \leq y_{2}$. In addition, we define $x_{1} \wedge x_{2}=\min \left\{x_{1}, x_{2}\right\}$ and $x_{1} \vee x_{2}=\max \left\{x_{1}, x_{2}\right\}$.

\section{UNIFORM ALLOCATION}

We first examine uniform allocation mechanism. Given the supplier's wholesale price and capacity level, we express the retailers' optimization problems as:

$$
\begin{aligned}
\tilde{\Pi}_{i}\left(r_{j}, w\right)= & \max _{r_{i} \leq K}\left\{\left(z_{i}-\tilde{q}_{i}\left(r_{i}, r_{j}\right)-\tilde{q}_{j}\left(r_{i}, r_{j}\right)\right.\right. \\
& \left.-w) \cdot \tilde{q}_{i}\left(r_{i}, r_{j}\right)\right\}, \\
= & \max _{r_{i} \leq K}\left\{\tilde{\Pi}_{i}^{1}=\max _{r_{i} \leq K-r_{j}}\left(z_{i}-w-r_{i}-r_{j}\right) r_{i},\right. \\
\tilde{\Pi}_{i}^{2}= & \max _{r_{i}>K-r_{j} ; r_{i} \leq r_{j}}\left(z_{i}-w-\left(r_{i} \wedge \frac{K}{2}\right)\right. \\
& \left.-\left(\left(K-r_{i}\right) \vee \frac{K}{2}\right)\right) \cdot\left(r_{i} \wedge \frac{K}{2}\right), \\
\tilde{\Pi}_{i}^{3}= & \max _{r_{i}>K-r_{j}, r_{i}>r_{j}}\left(z_{i}-w-\left(\left(K-r_{j}\right) \vee \frac{K}{2}\right)\right. \\
& \left.\left.-\left(r_{j} \wedge \frac{K}{2}\right)\right) \cdot\left(\left(K-r_{j}\right) \vee \frac{K}{2}\right)\right\},
\end{aligned}
$$

where $i, j=1,2$ and $i \neq j$. The following lemma characterizes the retailers' best response functions and associated profits.

LEMMA 1: Given retailer $j$ 's order quantity $r_{j}$, let $\tilde{r}_{i}\left(r_{j}\right)$ be retailer $i$ 's best response function $(i, j=1,2$ and $i \neq j)$. Define $\tilde{\alpha}_{i}=z_{i}-w-\sqrt{2 K\left(z_{i}-w-K\right)}$, we then have:

i. $w \in\left(0, z_{i}-2 K\right]$ : if $r_{j}<K / 2$, then $\tilde{r}_{i}\left(r_{j}\right) \in$ $\left[K-r_{j}, K\right]$ with $\tilde{\Pi}_{i}\left(r_{j}, w\right)=\left(z_{i}-w-K\right)\left(K-r_{j}\right)$; if $r_{j} \geq K / 2$, then $\tilde{r}_{i}\left(r_{j}\right) \in[K / 2, K]$ with $\tilde{\Pi}_{i}\left(r_{j}, w\right)=$ $\left(z_{i}-w-K\right) K / 2$.

ii. $w \in\left(z_{i}-2 K, z_{i}-3 K / 2\right.$ ]: if $r_{j} \leq 2 K-z_{i}+w$, then $\tilde{r}_{i}\left(r_{j}\right)=\left(z_{i}-w-r_{j}\right) / 2$ with $\tilde{\Pi}_{i}\left(r_{i}, w\right)=$ $\left(z_{i}-w-r_{j}\right)^{2} / 4$; if $2 K-z_{i}+w<r_{j} \leq K / 2$, then $\tilde{r}_{i}\left(r_{j}\right) \in\left[K-r_{j}, K\right]$ with $\tilde{\Pi}_{i}\left(r_{j}, w\right)=\left(z_{i}-w-\right.$
$K)\left(K-r_{j}\right)$; if $r_{j}>K / 2$, then $\tilde{r}_{i}\left(r_{j}\right) \in[K / 2, K]$ with $\tilde{\Pi}_{i}\left(r_{j}, w\right)=\left(z_{i}-w-K\right) K / 2$.

iii. $w \in\left(z_{i}-3 K / 2, z_{i}-K\right]$ : if $r_{j} \leq \tilde{\alpha}_{i}$, then $\tilde{r}_{i}\left(r_{j}\right)=$ $\left(z_{i}-w-r_{j}\right) / 2$ with $\tilde{\Pi}_{i}\left(r_{j}, w\right)=\left(z_{i}-w-r_{j}\right)^{2} / 4$;if $r_{j}>\tilde{\alpha}_{i}$, then $\tilde{r}_{i}\left(r_{j}\right) \in[K / 2, K]$ with $\tilde{\Pi}_{i}\left(r_{j}, w\right)=$ $\left(z_{i}-w-K\right) K / 2$.

iv. $w \in\left(z_{i}-K, z_{i}\right)$ : if $r_{j} \leq z_{i}-w$, then $\tilde{r}_{i}\left(r_{j}\right)=$ $\left(z_{i}-w-r_{j}\right) / 2$ with $\tilde{\Pi}_{i}\left(r_{j}, w\right)=\left(z_{i}-w-r_{j}\right)^{2} / 4 ;$ if $r_{j}>z_{i}-w$, then $\tilde{r}_{i}\left(r_{j}\right)=0$ with $\tilde{\Pi}_{i}\left(r_{j}, w\right)=0$.

The results show that a retailer's best response order quantity directly depends on the supplier's wholesale price and the other retailer's order size. In line with the definition of uniform allocation, a very intuitive observation is that retailer $i$ would always receive the same allocation $K / 2$ by ordering from $K / 2$ to $K$ (the maximum allowed order) if retailer $j$ 's order size is no less than $K / 2$. This explains the last part of (i), (ii), and (iii). However, if the wholesale price is very high, then the retailers cannot afford it and order nothing (see the second part of (iv)). Further, given an appropriate wholesale price, if the competitor orders a reasonably small quantity such that total capacity is sufficient for both retailers, then the best response function is the same as in the case without capacity limit. The first parts of (ii), (iii), and (iv) illustrate this effect. Conversely, from the second part of (i), we can see that if the wholesale price is very low, together with the fact that the other retailer's order quantity is less than $K / 2$, then retailer $i$ will order from $K-r_{2}$ to $K$ and gain the same resulting allocation $K-r_{2}$.

\subsection{Equilibrium Analysis under Uniform Allocation}

In this section, our objective is to characterize retailer order sizes in equilibrium and the optimal wholesale pricing for the supplier. Using backward induction, under uniform allocation, we first derive the retailers' equilibrium orders given any wholesale price, and then examine the supplier's wholesale pricing decisions.

The following theorem characterizes Nash equilibria of the retailers' order quantities.

THEOREM 1: Suppose the Nash equilibrium orders are $\left(\tilde{r}_{1}^{*}, \tilde{r}_{2}^{*}\right)$ under uniform mechanism. Define $\tilde{w}^{+}=2 z_{2}-z_{1}-$ $\frac{9}{4} K+\frac{3}{4} \sqrt{K^{2}+8 K\left(z_{1}-z_{2}\right)}$, then we have

(I) $K \leq z_{1}-z_{2}$ :

(i) if $w \in\left(0, z_{2}-3 K / 2\right]$, then any point in $[K / 2, K] \times[K / 2, K]$ is a Nash equilibrium;

(ii) if $w \in\left(z_{2}-3 K / 2, z_{2}-K\right]$, then any point in $\left[\tilde{\alpha}_{2}, K\right] \times[K / 2, K]$ is a Nash equilibrium;

(iii) if $w \in\left(z_{2}-K, z_{1}-2 K\right]$, then there exists a unique Nash equilibrium $\left(\tilde{r}_{1}^{*}, \tilde{r}_{2}^{*}\right)=(K, 0)$; 
(iv) if $w \in\left(z_{1}-2 K, z_{1}\right)$, then there exists a unique Nash equilibrium $\left(\tilde{r}_{1}^{*}, \tilde{r}_{2}^{*}\right)=\left(\left(z_{1}-\right.\right.$ w) $/ 2,0)$.

(II) $K>z_{1}-z_{2}$ :

(i) if $w \in\left(0, z_{2}-3 K / 2\right]$, then any point in $[K / 2, K] \times[K / 2, K]$ is a Nash equilibrium;

(ii) if $w \in\left(z_{2}-3 K / 2, \tilde{w}^{+}\right]$, then any point in $\left[\tilde{\alpha}_{2}, K\right] \times[K / 2, K]$ is a Nash equilibrium;

(iii) if $w \in\left(\tilde{w}^{+}, z_{1}-3 K / 2\right]$, then $\left(\tilde{r}_{1}^{*}, \tilde{r}_{2}^{*}\right)=$ $\left(\left(2 z_{1}-z_{2}-w\right) / 3,\left(2 z_{2}-z_{1}-w\right) / 3\right)$ is a Nash equilibrium and any point in $\left[\tilde{\alpha}_{2}, K\right] \times$ $[K / 2, K]$ is also a Nash equilibrium. Further, the former equilibrium dominates the latter by generating greater profits for both retailers;

(iv) if $w \in\left(z_{1}-3 K / 2, z_{2}-K\right]$, then $\left(\tilde{r}_{1}^{*}, \tilde{r}_{2}^{*}\right)=$ $\left(\left(2 z_{1}-z_{2}-w\right) / 3,\left(2 z_{2}-z_{1}-w\right) / 3\right)$ is a Nash equilibrium and any point in $\left[\tilde{\alpha}_{2}, K\right] \times$ $\left[\tilde{\alpha}_{1}, K\right]$ is also a Nash equilibrium. Further, the former equilibrium dominates the latter one by generating greater profits for both retailers;

(v) if $w \in\left(z_{2}-K, 2 z_{2}-z_{1}\right]$, then there exists a unique equilibrium $\left(\tilde{r}_{1}^{*}, \tilde{r}_{2}^{*}\right)=\left(\left(2 z_{1}-z_{2}-\right.\right.$ $\left.w) / 3,\left(2 z_{2}-z_{1}-w\right) / 3\right)$;

(vi) if $w \in\left(2 z_{2}-z_{1}, z_{1}\right)$, then there exists a unique equilibrium $\left(\tilde{r}_{1}^{*}, \tilde{r}_{2}^{*}\right)=\left(\left(z_{1}-\right.\right.$ $w) / 2,0)$.

Theorem 1 presents equilibrium orders when the two retailers compete for demand with different market powers. Equilibrium analysis is conducted for the cases $K \leq z_{1}-z_{2}$ and $K>z_{1}-z_{2}$ separately. The results are quite intuitive. First, when capacity is no greater than $z_{1}-z_{2}$, if the wholesale price is fairly low, with $w \in\left(0, z_{2}-K\right]$, then both retailers seek to gain higher allocations, but any one of them can receive only $K / 2$ by ordering no less than $K / 2$. Second, as the wholesale price increases, the low-type retailer cannot afford it and thus is driven out of the game. As a result, the problem reduces to a simple capacity allocation consisting of a single supplier and a single retailer. In this special case, the high-type retailer's profit function is $\tilde{\Pi}_{1}=\left(z_{1}-w-\tilde{q}_{1}\right) \tilde{q}_{1}$. Taking the first-order condition with respect to $\tilde{q}_{1}$, the optimal order follows that $\tilde{q}_{1}^{*}=\left(z_{1}-w\right) / 2$ without capacity constraint. Consequently, (a) if $w \leq z_{1}-2 K$, that is, $K \leq\left(z_{1}-w\right) / 2$, which implies that the capacity is limited for the retailer, then the equilibrium orders and the allocations are both $(K, 0)$; (b) if $w>z_{1}-2 K$, that is, $K>\frac{z_{1}-w}{2}$, where the capacity is sufficient to satisfy the retailer's order, then the equilibrium orders and allocations are both $\left(\left(z_{1}-w\right) / 2,0\right)$.

Moreover, when the capacity level exceeds the value $z_{1}-z_{2}$, certain results are similar to the case with $K \leq z_{1}-z_{2}$ when the wholesale price is very low or sufficiently high. We also find several other interesting results. In particular, when $w \in\left(z_{2}-3 K / 2, \tilde{w}^{+}\right]$, as $2 z_{2}-z_{1}-3 K / 2<\left(z_{1}+z_{2}-3 K\right) / 2$, it follows that if $2 q_{2}^{*}<q_{1}^{*}+q_{2}^{*}<K<K\left(\tilde{w}^{+}\right)$, where $K\left(\tilde{w}^{+}\right)=\left(z_{2}-w+\sqrt{\left(z_{2}-w\right)^{2}-8\left(2 z_{2}-z_{1}-w\right)^{2} / 9}\right) / 2$, then the low-type retailer would inflate her order size to be no less than $K / 2$ so as to gain more profit from the allocations $(K / 2, K / 2)$ than from the ideal allocations $\left(\left(2 z_{1}-\right.\right.$ $\left.\left.z_{2}-w\right) / 3,\left(2 z_{2}-z_{1}-w\right) / 3\right)$. This result reflects the fact that uniform mechanism has potential to favor the low-type retailer by offering an opportunity to diminish her competitive gap relative to the high-type retailer. Note that when $w \in\left(\tilde{w}^{+}, z_{2}-K\right]$, although there exist multiple equilibrium orders, the equilibrium $\left(\tilde{r}_{1}^{*}, \tilde{r}_{2}^{*}\right)=\left(\left(2 z_{1}-z_{2}-w\right) / 3,\left(2 z_{2}-\right.\right.$ $\left.z_{1}-w\right) / 3$ ) dominates other equilibria by generating more profits for both retailers.

Cho and Tang [6] and Liu [10] also study equilibrium ordering under uniform allocation. Cho and Tang [6], mostly study whether the gaming effect presents under uniform allocation and does not provide complete equilibrium orders under different conditions of $K$ and $w$. The analysis in Lemma 1 and Theorem 1 makes the following major distinctions comparing with [10]: (1) we assume an upper bound (supplier's capacity $K$ ) on order size, while Liu (2012) assumes no upper bound on order size; (2) Liu [10] assumes $z_{2}>\left(z_{1}+w\right) / 2$, while in our problem $w$ is a decision variable of the supplier, and it would be artificial to impose a lower bound on $w$ other than 0 , thus our article, relax the assumption as $z_{2}>z_{1} / 2$; (3) we provide complete and precise structures for retailers' ordering decisions in Nash and dominant equilibrium in Theorem 1, while Liu [10] focuses on allocations in stead of equilibrium orders and only provides a representative equilibrium when multiple equilibria result in the same allocation. In addition, Liu [10] classifies equilibria based on capacity level $K$, while we classify equilibria based on wholesale price $w$, for the ease of subsequent analysis of the supplier's wholesale pricing decision, which [10] does not consider. In fact, if we transform from $\tilde{w}(K)$ to $K(\tilde{w})$, it follows that $K=\left(z_{2}-w\right) / 2+$ $\left(\sqrt{-8 z_{1}^{2}+32 z_{1} z_{2}-23 z_{2}^{2}-16 z_{1} w+14 z_{1} w+14 z_{2} w+w^{2}}\right) / 6$, which is actually the threshold $K_{2}^{+}$used in [10]. Therefore, our results essentially are consistent with the results in [10], and are more complete from the perspective of equilibrium analysis.

\subsection{Supplier's Decisions under Uniform Allocation}

Now, we consider the supplier's decisions on wholesale price. By anticipating the retailers' best response order quantities and the allocations they will receive, the supplier chooses an optimal wholesale price to maximize his profit.

First, we establish the supplier's profit function. Note that when $K \leq z_{1}-z_{2}$, if $w \in\left(0, z_{1}-2 K\right]$, then the total 
Table 1. Supplier's pricing decisions and profits under uniform allocation

\begin{tabular}{|c|c|c|c|c|c|}
\hline K & $\left(0, \frac{z_{1}}{4}\right]$ & $\left(\frac{z_{1}}{4}, z_{1}-z_{2}\right]$ & $\left(z_{1}-z_{2}, \tilde{K}_{2}^{+}\right]$ & $\left(\tilde{K}_{2}^{+},+\infty\right)$ & - \\
\hline$\tilde{w}^{*}$ & $z_{1}-2 K$ & $z_{1} / 2$ & & $\underset{\in\left\{\tilde{w}^{+}, z_{1} / 2\right\}}{\operatorname{argmax}} \max \left\{K \tilde{w}^{+}, z_{1}^{2} / 8\right\}$ & $\frac{z_{1}}{2}<z_{2} \leq \frac{5 z_{1}}{7}$ \\
\hline$\tilde{\Pi}_{s}^{*}$ & $K\left(z_{1}-2 K\right)$ & $z_{1}^{2} / 8$ & & $\max \left\{K \tilde{w}^{+}, z_{1}^{2} / 8\right\}$ & \\
\hline$\tilde{w}^{*}$ & $z_{1}-2 K$ & $z_{1} / 2$ & $\underset{w \in\left\{\tilde{w}^{+}, z_{1} / 2\right\}}{\operatorname{argmax}} \max \left\{K \tilde{w}^{+}, z_{1}^{2} / 8\right\}$ & $\underset{w \in\left\{\tilde{w}^{+},\left(z_{1}+z_{2}\right) / 4, z_{1} / 2\right\}}{\operatorname{argmax}} \max \left\{K \tilde{w}^{+},\left(z_{1}+z_{2}\right)^{2} / 24, z_{1}^{2} / 8\right\}$ & $\frac{5 z_{1}}{7}<z_{2} \leq \frac{3 z_{1}}{4}$ \\
\hline$\tilde{\Pi}_{s}^{*}$ & $K\left(z_{1}-2 K\right)$ & $z_{1}^{2} / 8$ & $\max \left\{K \tilde{w}^{+}, z_{1}^{2} / 8\right\}$ & $\max \left\{K \tilde{w}^{+},\left(z_{1}+z_{2}\right)^{2} / 24, z_{1}^{2} / 8\right\}$ & \\
\hline$\tilde{w}^{*}$ & \multicolumn{2}{|c|}{$z_{1}-2 K$} & $\underset{w \in\left\{\tilde{w}^{+}, 2 z_{2}-z_{1}\right\}}{\operatorname{argmax}} \max \left\{K \tilde{w}^{+},\left(2 z_{2}-z_{1}\right)\left(z_{1}-z_{2}\right)\right\}$ & $\underset{w \in\left\{\tilde{w}^{+},\left(z_{1}+z_{2}\right) / 4,2 z_{2}-z_{1}\right\}}{\operatorname{argmax}} \max \left\{K \tilde{w}^{+},\left(z_{1}+z_{2}\right)^{2} / 24,\left(2 z_{2}-z_{1}\right)\left(z_{1}-z_{2}\right)\right\}$ & $\frac{3 z_{1}}{4}<z_{2}<z_{1}$ \\
\hline$\tilde{\Pi}_{s}^{*}$ & \multicolumn{2}{|c|}{$K\left(z_{1}-2 K\right)$} & $\max \left\{K \tilde{w}^{+},\left(2 z_{2}-z_{1}\right)\left(z_{1}-z_{2}\right)\right\}$ & $\max \left\{K \tilde{w}^{+},\left(z_{1}+z_{2}\right)^{2} / 24,\left(2 z_{2}-z_{1}\right)\left(z_{1}-z_{2}\right)\right\}$ & \\
\hline
\end{tabular}

allocated capacity is $K$; thus, in this scenario the supplier's profit is $K w$. Otherwise, if $w \in\left(z_{1}-2 K, z_{1}\right)$, then retailer 2 would be driven out of the market, and thus the supplier's profit would be $w\left(z_{1}-w\right) / 2$. Therefore, the supplier's maximum profit can be characterized as $\max \left\{\tilde{\Pi}_{s}^{1}, \tilde{\Pi}_{s}^{2}\right\}$, where

$$
\tilde{\Pi}_{s}^{1}=\max _{w \in\left(0, z_{1}-2 K\right]} K w, \quad \tilde{\Pi}_{s}^{2}=\max _{w \in\left(z_{1}-2 K, z_{1}\right)} \frac{w\left(z_{1}-w\right)}{2} .
$$

Similarly, when $K>z_{1}-z_{2}$, the supplier's problem is $\max _{w}\left\{\tilde{\Pi}_{s}^{3}, \tilde{\Pi}_{s}^{4}, \tilde{\Pi}_{s}^{5}\right\}$, where

$$
\begin{aligned}
& \tilde{\Pi}_{s}^{3}=\max _{w \in\left(0, \tilde{w}^{+}\right]} K w, \quad \tilde{\Pi}_{s}^{4}=\max _{w \in\left(\tilde{w}^{+}, 2 z_{2}-z_{1}\right]} \frac{w\left(z_{1}+z_{2}-2 w\right)}{3}, \\
& \tilde{\Pi}_{s}^{5}=\max _{w \in\left(2 z_{2}-z_{1}, z_{1}\right)} \frac{w\left(z_{1}-w\right)}{2} .
\end{aligned}
$$

To determine the optimal wholesale price $\tilde{w}^{*}$ and the corresponding profit $\tilde{\Pi}_{s}^{*}$ for the supplier, we need to compare $\tilde{\Pi}_{s}^{1}, \tilde{\Pi}_{s}^{2}$ and $\tilde{\Pi}_{s}^{3}, \tilde{\Pi}_{s}^{4}, \tilde{\Pi}_{s}^{5}$. The corresponding results are in Proposition 1 in the Appendix. We illustrate the supplier's optimal wholesale pricing decisions in Table 1, where the first row presents different levels of the capacity $K$ where a full range of $K>0$ is covered, and the last column denotes the competitive gap between the two retailers' market powers where a full range of $z_{1} / 2<z_{2}<z_{1}$ is covered. For any capacity level $K$ and competitive gap between $z_{1}$ and $z_{2}$, the optimal wholesale price $\tilde{w}^{*}$ and associated profit $\tilde{\Pi}_{s}^{*}$ are listed in the corresponding cells of the tables. We can see that under different conditions, the supplier can strategically alter the wholesale price to maximize his profit.

\section{PROPORTIONAL AND LEXICOGRAPHIC ALLOCATIONS}

First, we investigate proportional allocation. Under this mechanism, the retailers' optimization problem can be expressed as

$$
\begin{aligned}
\hat{\Pi}_{i}\left(r_{j}, w\right)= & \max _{r_{i} \leq K}\left\{\left(z_{i}-\hat{q}_{i}\left(r_{i}, r_{j}\right)\right.\right. \\
& \left.\left.-\hat{q}_{j}\left(r_{i}, r_{j}\right)-w\right) \cdot \hat{q}_{i}\left(r_{i}, r_{j}\right)\right\} \\
= & \max _{r_{i} \leq K}\left\{z_{i}-\left(\frac{K r_{i}}{r_{i}+r_{j}} \wedge r_{i}\right)\right. \\
& \left.-\left(\frac{K r_{j}}{r_{i}+r_{j}} \wedge r_{j}\right)-w\right\} \cdot\left(\frac{K r_{i}}{r_{i}+r_{j}} \wedge r_{i}\right),
\end{aligned}
$$

where $i, j=1,2$ and $i \neq j$.

Due to space limit, retailers' best response functions and equilibrium ordering decisions are provided in Lemma 2 and Proposition 2 in the Appendix. Using the same approach as for uniform allocation, we derive the supplier's optimal wholesale price and the associated maximum profit under proportional allocation, as shown in Table 2, in a similar structure as Table 1 . Table 2 shows that proportional allocation performs similar uniform allocation with respect to the supplier's pricing decision. Specifically, the supplier's capacity level and the competitive gap between the two retailers' market powers both directly affect the supplier's pricing decisions.

Now, we consider lexicographic allocation. Suppose priority is given to retailer $i$; then the retailers' profit function can be rewritten as:

$$
\begin{aligned}
\check{\Pi}_{i}\left(r_{j}, w\right)= & \max _{r_{i} \leq K}\left\{\left(z_{i}-w-\check{q}_{i}\left(r_{i}, r_{j}\right)\right.\right. \\
& \left.-\check{q}_{j}\left(r_{i}, r_{j}\right) \cdot \check{q}_{i}\left(r_{i}, r_{j}\right)\right\},
\end{aligned}
$$

Naval Research Logistics DOI 10.1002/nav 
Table 2. Supplier's pricing decisions and profits under proportional allocation

\begin{tabular}{|c|c|c|c|c|c|}
\hline K & $\left(0, \frac{z_{1}}{4}\right]$ & $\left(\frac{z_{1}}{4}, z_{1}-z_{2}\right]$ & $\left(z_{1}-z_{2}, \hat{K}_{2}^{+}\right]$ & $\left(\hat{K}_{2}^{+},+\infty\right)$ & - \\
\hline$\hat{w}^{*}$ & $z_{1}-2 K$ & $z_{1} / 2$ & & $\underset{\in\left\{\hat{w}^{-}, z_{1} / 2\right\}}{\operatorname{argmax}} \max \left\{K \hat{w}^{-}, z_{1}^{2} / 8\right\}$ & $\frac{z_{1}}{2}<z_{2} \leq \frac{5 z_{1}}{7}$ \\
\hline$\hat{\Pi}_{s}^{*}$ & $K\left(z_{1}-2 K\right)$ & $z_{1}^{2} / 8$ & & $\max \left\{K \hat{w}^{-}, z_{1}^{2} / 8\right\}$ & \\
\hline$\hat{w}^{*}$ & $z_{1}-2 K$ & $z_{1} / 2$ & $\underset{w \in\left\{\hat{w}^{-}, z_{1} / 2\right\}}{\operatorname{argmax}} \max \left\{K \hat{w}^{-}, z_{1}^{2} / 8\right\}$ & $\underset{w \in\left\{\hat{w}^{-},\left(z_{1}+z_{2}\right) / 4, z_{1} / 2\right\}}{\operatorname{argmax}} \max \left\{K \hat{w}^{-},\left(z_{1}+z_{2}\right)^{2} / 24, z_{1}^{2} / 8\right\}$ & $\frac{5 z_{1}}{7}<z_{2} \leq \frac{3 z_{1}}{4}$ \\
\hline$\hat{\Pi}_{s}^{*}$ & $K\left(z_{1}-2 K\right)$ & $z_{1}^{2} / 8$ & $\max \left\{K \hat{w}^{-}, z_{1}^{2} / 8\right\}$ & $\max \left\{K \hat{w}^{-},\left(z_{1}+z_{2}\right)^{2} / 24, z_{1}^{2} / 8\right\}$ & \\
\hline$\hat{w}^{*}$ & \multicolumn{2}{|c|}{$z_{1}-2 K$} & $\underset{w \in\left\{\hat{w}^{-}, 2 z_{2}-z_{1}\right\}}{\operatorname{argmax}} \max \left\{K \hat{w}^{-},\left(2 z_{2}-z_{1}\right)\left(z_{1}-z_{2}\right)\right\}$ & $\underset{w \in\left\{\hat{w}^{-},\left(z_{1}+z_{2}\right) / 4,2 z_{2}-z_{1}\right\}}{\operatorname{argmax}} \max \left\{K \hat{w}^{-},\left(z_{1}+z_{2}\right)^{2} / 24,\left(2 z_{2}-z_{1}\right)\left(z_{1}-z_{2}\right)\right\}$ & $\frac{3 z_{1}}{4}<z_{2}<z_{1}$ \\
\hline$\hat{\Pi}_{s}^{*}$ & \multicolumn{2}{|c|}{$K\left(z_{1}-2 K\right)$} & $\max \left\{K \hat{w}^{-},\left(2 z_{2}-z_{1}\right)\left(z_{1}-z_{2}\right)\right\}$ & $\max \left\{K \hat{w}^{-},\left(z_{1}+z_{2}\right)^{2} / 24,\left(2 z_{2}-z_{1}\right)\left(z_{1}-z_{2}\right)\right\}$ & \\
\hline
\end{tabular}

Table 3. Supplier's pricing decisions and profits under lexicographic allocation

\begin{tabular}{|c|c|c|c|c|c|}
\hline K & $\left(0, \frac{z_{1}}{4}\right]$ & $\left(\frac{z_{1}}{4}, z_{1}-z_{2}\right]$ & $\left(z_{1}-z_{2}, \check{K}_{i}^{+}\right]$ & $\left(\check{K}_{i}^{+},+\infty\right)$ & - \\
\hline$\check{w}^{*}$ & $z_{1}-2 K$ & $z_{1} / 2$ & & $\underset{\left\{\check{w}_{i}^{+}, z_{1} / 2\right\}}{\operatorname{rgmax}} \max \left\{K \check{w}_{i}^{+}, z_{1}^{2} / 8\right\}$ & $\frac{z_{1}}{2}<z_{2} \leq \frac{5 z_{1}}{7}$ \\
\hline$\check{\Pi}_{s}^{*}$ & $K\left(z_{1}-2 K\right)$ & $z_{1}^{2} / 8$ & & $\max \left\{K \check{w}_{i}^{+}, z_{1}^{2} / 8\right\}$ & \\
\hline$\check{w}^{*}$ & $z_{1}-2 K$ & $z_{1} / 2$ & $\underset{w \in\left\{\breve{w}_{i}^{+}, z_{1} / 2\right\}}{\operatorname{argmax}} \max \left\{K \check{w}_{i}^{+}, z_{1}^{2} / 8\right\}$ & $\underset{w \in\left\{\check{w}_{i}^{+},\left(z_{1}+z_{2}\right) / 4, z_{1} / 2\right\}}{\operatorname{argmax}} \max \left\{K \check{w}_{i}^{+},\left(z_{1}+z_{2}\right)^{2} / 24, z_{1}^{2} / 8\right\}$ & $\frac{5 z_{1}}{7}<z_{2} \leq \frac{3 z_{1}}{4}$ \\
\hline$\check{\Pi}_{s}^{*}$ & $K\left(z_{1}-2 K\right)$ & $z_{1}^{2} / 8$ & $\max \left\{K \check{w}_{i}^{+}, z_{1}^{2} / 8\right\}$ & $\max \left\{K \check{w}_{i}^{+},\left(z_{1}+z_{2}\right)^{2} / 24, z_{1}^{2} / 8\right\}$ & \\
\hline$\check{w}^{*}$ & \multicolumn{2}{|c|}{$z_{1}-2 K$} & $\underset{w \in\left\{\tilde{w}_{i}^{+}, 2 z_{2}-z_{1}\right\}}{\operatorname{argmax}} \max \left\{K \check{w}_{i}^{+},\left(2 z_{2}-z_{1}\right)\left(z_{1}-z_{2}\right)\right\}$ & $\underset{w \in\left\{\breve{w}_{i}^{+},\left(z_{1}+z_{2}\right) / 4,2 z_{2}-z_{1}\right\}}{\operatorname{argmax}} \max \left\{K \check{w}_{i}^{+},\left(z_{1}+z_{2}\right)^{2} / 24,\left(2 z_{2}-z_{1}\right)\left(z_{1}-z_{2}\right)\right\}$ & $\frac{3 z_{1}}{4}<z_{2}<z_{1}$ \\
\hline$\check{\Pi}_{s}^{*}$ & \multicolumn{2}{|c|}{$K\left(z_{1}-2 K\right)$} & $\max \left\{K \check{w}_{i}^{+},\left(2 z_{2}-z_{1}\right)\left(z_{1}-z_{2}\right)\right\}$ & $\max \left\{K \check{w}_{i}^{+},\left(z_{1}+z_{2}\right)^{2} / 24,\left(2 z_{2}-z_{1}\right)\left(z_{1}-z_{2}\right)\right\}$ & \\
\hline
\end{tabular}

$$
\begin{aligned}
= & \max _{r_{i} \leq K}\left\{\check{\Pi}_{i}^{1}=\max _{r_{i} \leq K-r_{j}}\left(z_{i}-r_{i}-r_{j}-w\right) \cdot r_{i},\right. \\
& \left.\check{\Pi}_{i}^{2}=\max _{r_{i}>K-r_{j}}\left(z_{i}-K-w\right) \cdot r_{i}\right\}, \\
\check{\Pi}_{j}\left(r_{i}, w\right)= & \max _{r_{j} \leq K}\left\{\left(z_{j}-\check{q}_{i}\left(r_{i}, r_{j}\right)\right.\right. \\
& \left.\left.-\check{q}_{j}\left(r_{i}, r_{j}\right)-w\right) \cdot \check{q}_{j}\left(r_{i}, r_{j}\right)\right\}, \\
= & \max _{r_{j} \leq K}\left\{\check{\Pi}_{j}^{1}=\max _{r_{j} \leq K-r_{i}}\left(z_{j}-w-r_{i}-r_{j}\right) \cdot r_{j},\right. \\
& \left.\check{\Pi}_{j}^{2}=\max _{r_{j}>K-r_{i}}\left(z_{j}-K-w\right) \cdot\left(K-r_{i}\right)\right\},
\end{aligned}
$$

supplier's wholesale pricing associated with the profits in Table 3.

\section{COMPARISONS OF THREE MECHANISMS}

We have investigated uniform, proportional, and lexicographic allocations with regard to how they affect the supplier's whole pricing decision in a duopoly model with demand competition. An interesting question is which of the three allocations is preferred by the supplier, the retailers and the supply chain when the total order size exceeds the available capacity. Because the supplier is the Stackelberg leader in the capacity allocation game, in this section, we first compare the performances of the three allocations from the perspective of the supplier, then from the perspectives of the retailers and the supply chain, respectively.

where $i, j=1,2$ and $i \neq j$.

Due to space limits, equilibrium analysis and the correspondent discussions for lexicographic allocation are provided in Proposition 3 in the Appendix. We only present the 
For notational convenience, for any given capacity level, we denote the maximum profit of the supplier obtained from uniform allocation, proportional allocation, and lexicographic allocation-1 (order priority to high-type retailer), and lexicographic allocation-2 (order priority to low-type retailer) by $U, P, L_{1}$, and $L_{2}$, respectively. To elaborate the comparisons of $U, P, L_{1}$, and $L_{2}$, we establish a benchmark by considering the case where the two retailers' market powers are symmetric. Let $z_{1}=z_{2}=z$. Comparing the supplier's profits between uniform and proportional allocation (Tables 1 and 2), we obtain the results as: (i) If $K<z / 3$, then the supplier's profits under uniform and proportional allocations are $\tilde{\Pi}_{s}^{*}=K(z-3 K / 2)$ and $\left.\hat{\Pi}_{s}^{*}=K[z-3(\sqrt{2}-1) K)\right]$, respectively. It is easy to check that $\hat{\Pi}_{s}^{*}>\tilde{\Pi}_{s}^{*}$, and therefore the latter allocation rule is a better choice for the supplier; and (ii) If $z / 3 \leq K<(2+\sqrt{2}) z / 6$, then recall that $\tilde{\Pi}_{s}^{*}=z^{2} / 6$ and $\hat{\Pi}_{s}^{*}=K(z-3 K / 2)$ (clearly, proportional allocation still outperforms uniform allocation, as $\hat{\Pi}_{s}^{*}>\tilde{\Pi}_{s}^{*}$ ); and (iii) If capacity is sufficiently large, then $\tilde{\Pi}_{s}^{*}=\hat{\Pi}_{s}^{*}=z^{2} / 6$, which implies that the two allocation mechanisms are indifferent in allocating capacity from the supplier's perspective. Hence, we conclude that proportional mechanism is better than uniform allocation, independent of the supplier's capacity level. Chen et al. [4] show that lexicographic allocation is better than proportional allocation for the supplier, through earning greater profit, in the symmetric case with $z_{1}=z_{2}$ and $L_{1}=L_{2}$. Hence, we have that $U \leq P \leq L_{2}=L_{1}$, not affected by the supplier's capacity level.

Now, we consider the case when the retailers' market powers are asymmetric with $z_{1}>z_{2}$. Note that we assume $z_{2}>z_{1} / 2$. Tables $1-3$ show that from the perspective of the supplier, it is indifferent among the three mechanisms when the capacity level is below the threshold level $z_{1}-z_{2}$. This result is intuitive. Within a limited capacity, the two retailers both order large quantities such that the supplier's capacity is fully sold. Consequently, the supplier maximizes his profit by charging the same wholesale price under all the three mechanisms.

However, when $K>z_{1}-z_{2}$, the problem becomes more complex. Take uniform and proportional mechanisms, for example. Observing the supplier's profit functions under the two allocations, since $\hat{w}^{-}>\tilde{w}^{+}$, the profits are different only when $w$ is in the interval $\left[\tilde{w}^{+}, \hat{w}^{-}\right]$. Recall that $\tilde{\Pi}_{s}=\left(z_{1}+z_{2}-2 w\right) w / 3$, and $\hat{\Pi}_{s}=K w$. To show $\tilde{\Pi}_{s}<\hat{\Pi}_{s}$, it suffices to show $\left(z_{1}+z_{2}-2 w\right) / 3<K$, which is equivalent to $w>\left(z_{1}+z_{2}-3 K\right) / 2$. When $w \in\left[\tilde{w}^{+}, \hat{w}^{-}\right]$, it is easy to verify that $\tilde{w}^{+}>\left(z_{1}+z_{2}-3 K\right) / 2$. Hence, this implies that proportional mechanism outperforms uniform allocation by generating more profit for the supplier. Equivalently, we may conclude that the supplier can sell more under proportional mechanism than under uniform mechanism at a given wholesale price in this problem setting. Similarly, it is not difficult to verify that for the supplier, lexicographic mechanism performs at least equally well as proportional mechanism.

Further, it is interesting to find that, for the supplier, lexicographic allocation that grants order priority to the hightype retailer outperforms the case that grants priority to the low-type retailer. We interpret this finding as follows. In general, as the wholesale price increases, retailers will shrink their order sizes, and consequently the supplier may have excess capacity. To earn more profit, the supplier would like his capacity to be fully sold with higher profit. When $w \in\left(0, \check{w}_{i}^{+}\right],(i=1,2)$, the retailer with order priority orders the entire capacity and there is no excess capacity. As $\breve{w}_{1}^{+}>\breve{w}_{2}^{+}$, the supplier can sell more when priority is given to the high-type retailer.

In summary, we have the following result.

THEOREM 2: $U \leq P \leq L_{2} \leq L_{1}$, for any capacity level.

We note that Lemma 3 and Remark 1 by [10] indicate the result $U \leq P$ in Theorem 2 . To verify our findings and gain further insight, we conduct numerical studies, as shown in the Appendix. On examining the results, we have the following observations. First, when the supplier's capacity is relatively small or sufficiently large, the supplier is indifferent among the three allocation mechanisms. Second, the supplier's optimal wholesale price is not necessarily decreasing with the capacity level, and the associated profit is not necessarily increasing with the capacity level. Third, lexicographic allocation (especially when priority is given to the high-type retailer) can be evidently more profitable for the supplier than uniform and proportional allocations for any given capacity level and competitive gap. Fourth, the advantage of the superior allocation in each comparison becomes more obvious as downstream demand competition grows.

Now, we compare the total retailer and total supply chain profits under the three allocation mechanisms, respectively.

THEOREM 3: Let $\tilde{\Pi}_{r}^{*}, \hat{\Pi}_{r}^{*}$ and $\check{\Pi}_{r i}^{*}(i=1,2)$ be the total retailer profits, and $\tilde{\Pi}_{s c}^{*}, \hat{\Pi}_{s c}^{*}$ and $\check{\Pi}_{s c i}^{*}(i=1,2)$ be the total supply chain profits under uniform, proportional and lexicographic (with order priority giving to retailer $i$ ) mechanisms, respectively. We have

(I) $K \leq z_{1}-z_{2}$ :

(i) When $w \leq z_{2}-K, \check{\Pi}_{r 2}^{*} \leq \tilde{\Pi}_{r}^{*}=\hat{\Pi}_{r}^{*} \leq \check{\Pi}_{r 1}^{*}$ and $\check{\Pi}_{s c 2}^{*} \leq \tilde{\Pi}_{s c}^{*}=\hat{\Pi}_{s c}^{*} \leq \check{\Pi}_{s c 1}^{*}$.

(ii) When $w>z_{2}-K, \tilde{\Pi}_{r}^{*}=\hat{\Pi}_{r}^{*}=\check{\Pi}_{r 2}^{*}=\check{\Pi}_{r 1}^{*}$ and $\tilde{\Pi}_{s c}^{*}=\hat{\Pi}_{s c}^{*}=\check{\Pi}_{s c 2}^{*}=\check{\Pi}_{s c 1}^{*}$.

(II) $K>z_{1}-z_{2}$ :

(i) When $w \leq \tilde{w}^{+}, \check{\Pi}_{r 2}^{*} \leq \tilde{\Pi}_{r}^{*}=\hat{\Pi}_{r}^{*} \leq \check{\Pi}_{r 1}^{*}$ and $\check{\Pi}_{s c 2}^{*} \leq \tilde{\Pi}_{s c}^{*}=\hat{\Pi}_{s c}^{*} \leq \check{\Pi}_{s c 1}^{*}$.

(ii) When $\tilde{w}^{+}<w \leq \hat{w}^{-}, \tilde{\Pi}_{r}^{*} \geq \hat{\Pi}_{r}^{*}, \check{\Pi}_{r 1}^{*} \geq$ $\hat{\Pi}_{r}^{*} \geq \check{\Pi}_{r 2}^{*}, \check{\Pi}_{s c 1}^{*} \geq \hat{\Pi}_{s c}^{*} \geq \check{\Pi}_{s c 2}^{*} ; \check{\Pi}_{r 1}^{*} \geq \tilde{\Pi}_{r}^{*}$

Naval Research Logistics DOI 10.1002/nav 
if and only if $2 w^{2}-2\left(z_{1}+z_{2}-K\right) w+$ $5 z_{1}^{2}+5 z_{2}^{2}-8 z_{1} z_{2}-9\left(z_{1}-K\right) K \leq 0$; and $\check{\Pi}_{s c 1}^{*} \geq \tilde{\Pi}_{s c}^{*}$ if and only if $4 w^{2}-\left(z_{1}+z_{2}\right) w-$ $5\left(z_{1}^{2}+z_{2}^{2}\right)+8 z_{1} z_{2}+\frac{9}{2} K\left(z_{1}-K\right) \geq 0$.

(iii) When $\hat{w}^{-}<w \leq \check{w}_{2}^{+}, \tilde{\Pi}_{r}^{*}=\hat{\Pi}_{r}^{*}$, $\tilde{\Pi}_{s c}^{*}=$ $\hat{\Pi}_{s c}^{*} ; \check{\Pi}_{r i}^{*} \geq \tilde{\Pi}_{r}^{*}\left(\hat{\Pi}_{r}^{*}\right)$ if and only if $2 w^{2}-$ $2\left(z_{i}+z_{j}-K\right) w+5\left(z_{i}^{2}+z_{j}^{2}-K\right)-9\left(z_{i}-\right.$ $K) K \leq 0$; and $\check{\Pi}_{s c i}^{*} \geq \tilde{\Pi}_{s c}^{*}\left(\hat{\Pi}_{s c}^{*}\right)$ if and only if $4 w^{2}-\left(z_{i}+z_{j}\right) w-5\left(z_{i}^{2}+z_{j}^{2}\right)+8 z_{i} z_{j}+$ $\frac{9}{2} K\left(z_{i}-K\right) \geq 0$.

(iv) When $\check{w}_{2}^{+}<w \leq \check{w}_{1}^{+}, \tilde{\Pi}_{r}^{*}=\hat{\Pi}_{r}^{*}=\check{\Pi}_{r 2}^{*}$, $\check{\Pi}_{s c 2}^{*}=\tilde{\Pi}_{s c}^{*}=\hat{\Pi}_{s c}^{*} ; \check{\Pi}_{r 1}^{*} \geq \tilde{\Pi}_{r}^{*}\left(\hat{\Pi}_{r}^{*}, \check{\Pi}_{r 2}^{*}\right)$ if and only if $2 w^{2}-2\left(z_{1}+z_{2}-K\right) w+$ $5 z_{1}^{2}+5 z_{2}^{2}-8 z_{1} z_{2}-9\left(z_{1}-K\right) K \leq 0$; and $\check{\Pi}_{s c 1}^{*} \geq \tilde{\Pi}_{s c}^{*}\left(\hat{\Pi}_{s c}^{*}, \check{\Pi}_{r 2}^{*}\right)$ if and only if $4 w^{2}-\left(z_{1}+z_{2}\right) w-5\left(z_{1}^{2}+z_{2}^{2}\right)+8 z_{1} z_{2}+$ $\frac{9}{2} K\left(z_{1}-K\right) \geq 0$.

(v) When $w>\check{w}_{1}^{+}, \tilde{\Pi}_{r}^{*}=\hat{\Pi}_{r}^{*}=\check{\Pi}_{r 2}^{*}=\check{\Pi}_{r 1}^{*}$ and $\tilde{\Pi}_{s c}^{*}=\hat{\Pi}_{s c}^{*}=\check{\Pi}_{s c 2}^{*}=\check{\Pi}_{s c 1}^{*}$.

The first part of Theorem 3 has intuitive explanations. When $K \leq z_{1}-z_{2}$, if the wholesale price is sufficiently low, then each retailer will order as much as possible for her best interest. Under uniform and proportional allocations, the Nash equilibrium orders are $(K, K)$, resulting in allocations $(K / 2, K / 2)$, that is, the two retailers have the same profit. While under lexicographic allocation, the retailer with higher order priory will obtain the entire capacity $K$. As the retailer with greater market power can sell with a higher retail price, with allocation $K$ she can earn more profit than the total retailer profit under the other two mechanisms. Therefore, the total retailer profit is the highest under lexicographic allocation with order priority to the high-type retailer. Note that the total allocated capacity is $K$ under all the three allocations, and thus the total supply chain profit is also the highest under lexicographic allocation with order priority to the hightype retailer. Conversely, if the wholesale price is sufficiently high, then each retailer has no incentive to order more than her ideal allocation. Thus, the three allocation mechanisms result in the same total retailer profit and total supply chain profit. When $K>z_{1}-z_{2}$ and $w$ is neither very low nor very high, lexicographic allocation may bring the retailers and the supply chain either more or less profit than the other two mechanisms.

\section{EXTENSIONS: AN ARBITRARY NUMBER OF RETAILERS}

A key finding in earlier sections is the analytical characterization on the supplier's preference ranking of capacity allocation rules with two competing retailers, as in Theorem
2: (1) lexicographic with priority to high-type retailer; (2) lexicographic with priority to low-type retailer; (3) proportional; and (4) uniform. An interesting question is whether the finding still holds with an arbitrary number of retailers. We precede to answer the question in this section.

Suppose there are $n(n \geq 3)$ retailers with market power vector $z=\left(z_{1}, z_{2}, \ldots, z_{n}\right)$. Without loss of generality, we assume $z_{1} \geq z_{2} \geq \cdots \geq z_{n}$. Suppose that retailer $i$ with order quantity $r_{i}$ receives allocation $q_{i}, i=$ $1,2, \ldots, n$. Also, for notational convenience, let $r_{-i}=$ $\left(r_{1}, r_{2}, \ldots, r_{i-1}, r_{i+1}, \ldots, r_{n}\right), R_{-i}=\sum_{j=1, j \neq i}^{n} r_{j}, q_{-i}=$ $\left(q_{1}, q_{2}, \ldots, q_{i-1}, q_{i+1}, \ldots, q_{n}\right)$, and $Q_{-i}=\sum_{j=1, j \neq i}^{n} q_{j}$.

Similar to the case with two competing retailers, without capacity constraint, from retailer $i$ 's profit function $\Pi_{i}\left(r_{i}, r_{-i}\right)=\left(z_{i}-w-r_{i}-R_{-i}\right) r_{i}, i=1,2, \ldots, n$, we can obtain the equilibrium order quantity for each retailer $r_{i}^{*}=$ $\left((n+1) z_{i}-\sum_{j=1}^{n} z_{j}-w\right) /(n+1)$. In this case, every retailer's order will be satisfied with ideal allocation $q_{i}^{*}=r_{i}^{*}$, and the total allocated capacity is $Q^{*}=\left(\sum_{j=1}^{n} z_{j}-n w\right) /(n+1)$.

In Section 2, we describe uniform, proportional, and lexicographic allocation rules for the case with two competing retailers, which can be easily extended to the case with $n$ retailers and readers can refer to [7] for more details. Our objective is to find the supplier's preference of these rules. We next achieve this objective through comparison of total allocated capacity: for any given capacity $K$ and wholesale price $w$, the more the supplier sells under a mechanism, the better the mechanism performs from the perspective of the supplier.

Under all the three allocation mechanisms considered, our previous analysis shows two intuitive results regarding the total allocated capacity with a given wholesale price: (i) if the supplier's capacity level is sufficiently low, then the total order quantity exceeds the capacity and the total allocated capacity is $K$; (ii) if the supplier's capacity is sufficiently high, then every retailer will order her ideal allocation and the total allocated capacity is $Q^{*}$. That is, when the supplier's capacity level is either too low or too high, the three allocation mechanisms perform the same for the supplier.

However, when the supplier's capacity is at a medium level, which can be even sufficient to supply the total ideal allocation, that is, $K \geq Q^{*}$, the three allocation mechanisms provide different incentives for retailers with demand competition to inflate their order quantities to obtain more than ideal allocation. Specifically, under each allocation mechanism, there is a threshold $K^{*}$ such that, (i) if $K<K^{*}$, then the total allocated capacity is $K$; (ii) if $K \geq K^{*}$, then the total allocated capacity is $Q^{*}$. Because of order inflation, we have $Q^{*} \leq K^{*}$. Observation that for any given capacity $K$ and wholesale price $w$, the larger the threshold $K^{*}$ under an allocation mechanism, the more the supplier can sell under the mechanism. Let the thresholds under uniform, proportional and lexicographic mechanisms be $K_{u}^{*}, K_{p}^{*}$, and $K_{l_{t}}^{*}$, 
respectively, where $t \in\{1,2, \ldots, n !\}$ denotes one permutation, that is, priory sequence, of the $n$ retailers. Specifically, let $l^{*}$ denote the priority sequence $1 \rightarrow 2 \rightarrow \ldots n$, that is, the sequence of retailers in nonincreasing market powers.

THEOREM 4: For any wholesale price $w$, we have $K_{u}^{*} \leq$ $K_{p}^{*} \leq K_{l^{*}}^{*}$.

Theorem 4 means that the lexicographic allocation with priority sequence of retailers in nonincreasing market powers dominates proportional allocation, and proportional allocation dominates uniform allocation, from the perspective of the supplier, for any number of retailers.

Analytical results for lexicographic allocation with all sequences are more difficult to obtain as there are $n$ ! priority sequences of $n$ retailers that can be used. Next, we compare the performance of the three allocation mechanisms through numerical study, in terms of threshold $K^{*}$. Note that it is not only hard to find closed-form for $K^{*}$, but also hard to compute $K^{*}$ numerically. We provide in the Appendix a method for computing $K^{*}$ for the considered mechanisms. To compare all the priority sequences used by lexicographic allocation, we consider $n=3$, with $\left(z_{1}, z_{2}, z_{3}\right)=(100,85,80)$. There are $3 !=6$ priority sequences of the three retailers can be used under lexicographic allocation, denoted $l_{1}=$ $(80,85,100), l_{2}=(80,100,85), l_{3}=(85,80,100), l_{4}=$ $(85,100,80), l_{5}=(100,80,85), l_{6}=(100,85,80)$, respectively. To ensure that each retailer's ideal allocation is positive, that is, $q_{i}^{*}=r_{i}^{*}=\left((n+1) z_{i}-\sum_{j=1}^{n} z_{j}-w\right) /(n+1)>0$, $i=1,2,3$, we consider $w \leq 55$.

For wholesale prices $w=5,10, \ldots, 55$, Table 4 summarizes the results of the thresholds $K^{*}$ under different mechanisms. Consistent with Theorem 4 , we have $K_{p}^{*}>K_{u}^{*}$ for any $w$ considered. Also, we see $K_{l}^{*}>K_{u}^{*}$ for any wholesale price considered and any priority sequence of retailers, consistent with Theorem 2, theoretical result for the case with two retailers. In addition, we have $K_{l_{1}}^{*}<K_{p}^{*}$ when $w \leq 40, K_{l_{1}}^{*}>K_{p}^{*}$ when $w \geq 45$, and $K_{l_{t}}^{*}>K_{p}^{*}$ for any $w$, for $t=2, \ldots, 6$. That is, with three or more retailers, the relative performance of lexicographic allocation compared with proportional allocation depends on priority sequence of retailers: lexicographic allocation may outperform (underperform, resp.) proportional allocation if priority is given to retailers with high (low, resp.) market powers.

For a clearer view of the relative performance of different mechanisms, we directly compare the allocated capacities in Fig. 1. The total allocated capacities under uniform, proportional and lexicographic (with priority sequence $l_{6}$ ) mechanisms are depicted by lines O-A-D-G $\left(Q_{u}^{*}\right)$, O-B-E-G $\left(Q_{p}^{*}\right)$, O-C-F-G $\left(Q_{l_{6}}^{*}\right)$, respectively. Conversely, under all mechanisms, when the capacity is below the respective threshold $K^{*}$, the suppliers' total selling quantity is equal to the available capacity $K$. Conversely, when the capacity level exceeds
Table 4. Thresholds $K^{*}$ under different allocations, $n=3$

\begin{tabular}{lcccccccc}
\hline$w$ & $K_{u}^{*}$ & $K_{p}^{*}$ & $K_{l_{1}}^{*}$ & $K_{l_{2}}^{*}$ & $K_{l_{3}}^{*}$ & $K_{l_{4}}^{*}$ & $K_{l_{5}}^{*}$ & $K_{l_{6}}^{*}$ \\
\hline 5 & 69.20 & 75.43 & 75.11 & 79.16 & 75.97 & 77.36 & 82.15 & 82.15 \\
10 & 65.22 & 70.88 & 70.55 & 74.58 & 71.30 & 72.71 & 77.38 & 77.38 \\
15 & 61.22 & 66.32 & 65.99 & 70.01 & 66.63 & 68.03 & 72.61 & 72.61 \\
20 & 57.20 & 61.73 & 61.41 & 65.42 & 61.95 & 63.33 & 67.82 & 67.82 \\
25 & 53.16 & 57.13 & 56.84 & 60.82 & 57.28 & 58.60 & 63.00 & 63.00 \\
30 & 49.10 & 52.51 & 52.25 & 56.21 & 52.60 & 53.81 & 58.16 & 58.16 \\
35 & 45.01 & 47.84 & 47.66 & 51.59 & 47.92 & 48.96 & 53.27 & 53.27 \\
40 & 40.88 & 43.14 & 43.06 & 46.94 & 43.23 & 44.00 & 48.33 & 48.33 \\
45 & 36.72 & 38.37 & 38.44 & 42.28 & 38.55 & 38.86 & 43.32 & 43.32 \\
50 & 32.51 & 33.51 & 33.80 & 37.57 & 33.85 & 33.85 & 38.17 & 38.17 \\
55 & 28.23 & 28.51 & 29.15 & 32.81 & 29.15 & 29.15 & 32.81 & 32.81 \\
\hline
\end{tabular}

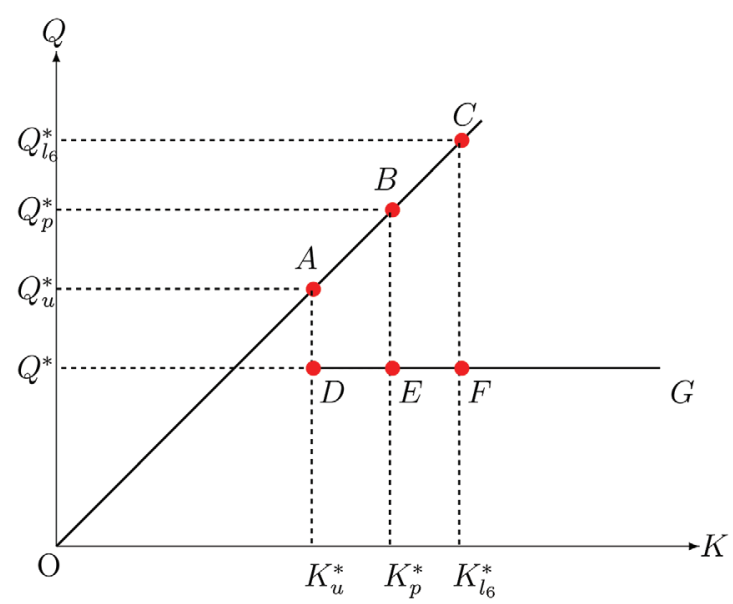

Figure 1. Total Allocated Capacities under Different Mechanisms, as Capacity Changes. [Color figure can be viewed at wileyonlinelibrary.com]

the respective threshold $K^{*}$, the total selling quantity is equal to $Q^{*}$.

Our numerical studies show that with more retailers, there exist more priority sequences with which proportional mechanism outperforms lexicographic mechanism. For example, with $n=5$ with $\left(z_{1}, z_{2}, z_{3}, z_{4}, z_{5}\right)=(100,99,98,97,96)$, results with lexicographic allocation with priority sequences $l_{1}=(96,97,98,99,100), l_{2}=(97,96,98,99,100), l_{3}=$ $(98,96,97,99,100), l_{4}=(99,96,97,98,100)$, and $l_{5}=$ $(100,99,98,97,96)$ are summarized in Table 5 , where proportional mechanism outperforms lexicographic mechanism with priority sequences $l_{1}, l_{2}$ and $l_{3}$. Note that lexicographic mechanism performs better for the supplier when giving higher priority to retailers with higher market power. When there are more retailers, the gap between retailers' market powers is relatively large and lexicographic mechanism becomes less efficient when giving higher priority to retailers with lower market power, which makes the mechanism outperformed by proportional mechanism with $n \geq 3$.

Next, we compare the total retailer and total supply chain profits under the three allocation mechanisms, respectively. 
Table 5. Thresholds $K^{*}$ under different allocations, $n=5$

\begin{tabular}{cccccccc}
\hline$w$ & $K_{u}^{*}$ & $K_{p}^{*}$ & $K_{l_{1}}^{*}$ & $K_{l_{2}}^{*}$ & $K_{l_{3}}^{*}$ & $K_{l_{4}}^{*}$ & $K_{l_{5}}^{*}$ \\
\hline 10 & 75.96 & 84.51 & 84.40 & 84.80 & 85.49 & 86.16 & 86.80 \\
20 & 67.52 & 75.01 & 74.45 & 75.09 & 75.77 & 76.44 & 77.09 \\
30 & 59.05 & 65.49 & 65.18 & 65.37 & 66.06 & 66.73 & 67.37 \\
40 & 50.55 & 55.98 & 55.63 & 55.66 & 56.35 & 57.01 & 57.64 \\
50 & 42.01 & 46.46 & 46.07 & 46.07 & 46.63 & 47.29 & 47.92 \\
60 & 33.41 & 36.94 & 36.51 & 36.51 & 36.92 & 37.57 & 38.19 \\
70 & 24.71 & 27.39 & 27.00 & 27.00 & 27.20 & 27.85 & 28.44 \\
80 & 16.01 & 17.81 & 17.63 & 17.63 & 17.63 & 18.12 & 18.67 \\
\hline
\end{tabular}

THEOREM 5: Let $\tilde{\Pi}_{r}^{*}, \hat{\Pi}_{r}^{*}$ and $\check{\Pi}_{r}^{*}$ be the total retailer profits, and $\tilde{\Pi}_{s c}^{*}, \hat{\Pi}_{s c}^{*}$ and $\check{\Pi}_{s c}^{*}$ be the total supply chain profits under uniform, proportional and lexicographic (with priority sequence of retailers nonincreasing in market power) mechanisms. We have

(i) When $K \leq K_{u}^{*}$, $\tilde{\Pi}_{r}^{*}=\hat{\Pi}_{r}^{*} \leq \check{\Pi}_{r}^{*}$ and $\tilde{\Pi}_{s c}^{*}=\hat{\Pi}_{s c}^{*} \leq$ $\check{\Pi}_{s c}^{*}$.

(ii) When $K_{u}^{*}<K \leq K_{p}^{*}, \tilde{\Pi}_{r}^{*} \geq \hat{\Pi}_{r}^{*}, \check{\Pi}_{r}^{*} \geq \hat{\Pi}_{r}^{*}$, $\check{\Pi}_{s c}^{*} \geq \hat{\Pi}_{s c}^{*} ; \check{\Pi}_{r}^{*} \geq \tilde{\Pi}_{r}^{*}$ if and only if $K^{2}-\left(z_{1}-\right.$ w) $K+\sum_{i=1}^{n}\left(z_{i}-w-Q^{*}\right) q_{i}^{*} \geq 0$; and $\check{\Pi}_{s c}^{*} \geq \tilde{\Pi}_{s c}^{*}$ if and only if $K^{2}-z_{1} K+\sum_{i=1}^{n}\left(z_{i}-Q^{*}\right) q_{i}^{*} \geq 0$.

(iii) When $K_{p}^{*}<K \leq K_{l *}^{*}, \tilde{\Pi}_{r}^{*}=\hat{\Pi}_{r}^{*}, \tilde{\Pi}_{s c}^{*}=\hat{\Pi}_{s c}^{*}$; $\check{\Pi}_{r}^{*} \geq \tilde{\Pi}_{r}^{*}\left(\hat{\Pi}_{r}^{*}\right)$ if and only if $K^{2}-\left(z_{1}-w\right) K+$ $\sum_{i=1}^{n}\left(z_{i}-w-Q^{*}\right) q_{i}^{*} \geq 0$; and $\check{\Pi}_{s c}^{*} \geq \tilde{\Pi}_{s c}^{*}\left(\hat{\Pi}_{s c}^{*}\right)$ if and only if $K^{2}-z_{1} K+\sum_{i=1}^{n}\left(z_{i}-Q^{*}\right) q_{i}^{*} \geq 0$.

(iv) When $K>K_{l *}^{*}, \tilde{\Pi}_{r}^{*}=\hat{\Pi}_{r}^{*}=\check{\Pi}_{r}^{*}$ and $\tilde{\Pi}_{s c}^{*}=\hat{\Pi}_{s c}^{*}=$ $\check{\Pi}_{s c}^{*}$.

Similar to the case with two retailers, for any wholesale price, when capacity level is sufficiently low, lexicographic mechanism with priority sequence of retailers nonincreasing in market power generates the highest total profit for the retailers and the supply chain among the three mechanisms. On the other extreme, if the capacity level is sufficiently high, then the three mechanisms perform the same. When the capacity is at a medium level, whether lexicographic mechanism with priority sequence of retailers nonincreasing in market power dominates the other two mechanisms depends on conditions specified in Theorem 5.

\section{CONCLUSIONS}

In the practice of production and operations, capacity allocation is an important problem when retailer total order quantity exceeds supplier available capacity. This article analyzes three capacity allocation mechanisms, uniform, proportional, and lexicographic allocations, in the presence of demand competition between retailers in a two-echelon decentralized supply chain. We consider the supplier's wholesale pricing decision together with his choice of allocation mechanisms, for any given capacity level. The pricing decision is important, as with exogenously given scarce capacity and the supplier's anticipation of retailers' ordering behavior, it can be more or profitable for the supplier to adjust his wholesale price.

From a modeling perspective, we consider both horizontal and vertical interactions between the supplier and two retailers. Specifically, in the horizontal interaction, the two competing retailers compete for demand from the same group of customers and the supplier's capacity simultaneously. Their desired orders are determined by Cournot competition under complete information. In the vertical interaction, the supplier acts as a leader who first announces his capacity, wholesale price, and an allocation rule with upper bound restriction on order size. Then, the retailers determine their order quantities. Finally, capacity is allocated and market demand is realized. Our analysis focuses particularly on the impact of allocation mechanisms on supplier pricing decisions and retailer order behavior.

Our results show that there may exist multiple Nash equilibria as the wholesale price changes under each allocation mechanism considered. Via equilibrium analysis, we identify exact conditions under which the gaming effect is present. Also, in our model, equilibrium orders are guaranteed under all mechanisms considered. This allows us to exactly compare the three mechanisms at any capacity level with regard to the supplier, the retailers and the supply chain.

From the perspective of the supplier, and in the asymmetric case, we show that when the capacity level is either very low or sufficiently high, the supplier is indifferent among the three mechanisms. The result is intuitive. When capacity is very small, retailers order large quantities and the supplier's capacity is fully sold under all mechanisms considered. As a result, the supplier can sell the total capacity $K$ by charging the same reasonably high wholesale price under all three mechanisms. Conversely, when the capacity level is sufficiently high, each retailer would like to order her ideal order size as if capacity is unlimited. However, for an intermediate range in capacity level, the supplier can sell more from lexicographic allocation than from uniform or proportional allocation, especially when giving order priority to the high-type retailer. Our further numerical studies verify this finding. We find that the advantage of lexicographic allocation becomes more obvious as the low-type retailer's market power is closer to that of the high-type retailer.

For the case with three or more retailers, we analytically prove that proportional allocation dominates uniform allocation and lexicographic allocation with priority sequence of retailers in nonincreasing market powers dominates proportional allocation from the perspective of the supplier. Also, we numerically show that lexicographic allocation with any 
priority sequence of retailers dominates uniform allocation for any number of retailers. With three or more retailers, the relative performance of lexicographic allocation compared with proportional allocation depends on priority sequence of retailers: lexicographic allocation may outperform (underperform, respectively) proportional allocation if priority is given to retailers with higher (lower, respectively) market powers. Further, we compare the mechanisms from the perspective of the retailers and the supply chain. We hope that these findings can provide a reference for suppliers in their selection of allocation mechanism and for their efficient pricing decisions.

In a two-echelon supply chain with a single supplier and multiple competing retailers, this article examines the impact of capacity allocation mechanisms on supplier pricing decisions and retailer ordering behavior. This model provides a foundation for future studies, for example, extending the supply chain to be more general, for example, some retailers have an alternative supplier or multiple suppliers with multiple retailers. Also, it is important to study how the supplier plans production if the down stream retailers face uncertainty of market demand. Further, incorporation of other allocation mechanisms, different kinds of market demand competition, and risk aversion for supply chain members, are interesting research questions.

\section{APPENDIX}

PROOF OF LEMMA 1: Because the ordering decisions are symmetric between the two retailers, to obtain retailer $i$ 's best response function $r_{i}\left(r_{j}\right)$, we only need to consider retailer 1's optimal problem:

$$
\begin{aligned}
& \tilde{\Pi}_{1}\left(r_{2}, w\right)=\max _{r_{1} \in[0, K]}\left\{\max _{r_{1} \leq K-r_{2}} \tilde{\Pi}_{1}^{1}, \quad \max _{r_{1}>K-r_{2} ; r_{1} \leq r_{2} ; r_{1}<\frac{K}{2}} \tilde{\Pi}_{1}^{2,1},\right. \\
& \max _{r_{1}>K-r_{2} ; r_{1} \leq r_{2} ; r_{1} \geq \frac{K}{2}} \tilde{\Pi}_{1}^{2,2}, \quad \max _{r_{1}>K-r_{2}, r_{1}>r_{2} ; r_{2}<\frac{K}{2}} \tilde{\Pi}_{1}^{3,1}, \\
& \left.\max _{r_{1}>K-r_{2}, r_{1}>r_{2} ; r_{2} \geq \frac{K}{2}} \tilde{\Pi}_{1}^{3,2}\right\},
\end{aligned}
$$

where $\quad \tilde{\Pi}_{1}^{1}=\left(z_{1}-w-r_{1}-r_{2}\right) \cdot r_{1} ; \quad \tilde{\Pi}_{1}^{2,1}=\left(z_{1}-w-K\right) \cdot r_{1} ; \quad \tilde{\Pi}_{1}^{2,2}=\left(z_{1}-w-K\right) \cdot \frac{K}{2} ;$ $\tilde{\Pi}_{1}^{3,1}=\left(z_{1}-w-K\right) \cdot\left(K-r_{2}\right) ; \quad \tilde{\Pi}_{1}^{3,2}=\left(z_{1}-w-K\right) \cdot \frac{K}{2}$.

The five subproblems can be solved as follows:

$$
\begin{gathered}
\tilde{r}_{1}^{1 *}=\underset{r_{1} \in\left[0, K-r_{2}\right]}{\arg \max } \tilde{\Pi}_{1}^{1}= \begin{cases}0, & \text { if } \frac{z_{1}-w-r_{2}}{2}<0, \\
\frac{z_{1}-w-r_{2}}{2}, & \text { if } 0 \leq \frac{z_{1}-w-r_{2}}{2} \leq K-r_{2}, \\
K-r_{2}, & \text { if } \frac{z_{1}-w-r_{2}}{2}>K-r_{2} .\end{cases} \\
\tilde{\Pi}_{1}^{1 *}=\max _{r_{1} \in\left[0, K-r_{2}\right]} \tilde{\Pi}_{1}^{1}= \begin{cases}0, & \text { if } \frac{z_{1}-w-r_{2}}{2}<0, \\
\frac{\left(z_{1}-w-r_{2}\right)^{2}}{4}, & \text { if } 0 \leq \frac{z_{1}-w-r_{2}}{2} \leq K-r_{2}, \\
\left(z_{1}-w-K\right)\left(K-r_{2}\right), & \text { if } \frac{z_{1}-w-r_{2}}{2}>K-r_{2} .\end{cases}
\end{gathered}
$$

$$
\begin{aligned}
\tilde{r}_{1}^{23 *}= & \underset{r_{1} \in\left(K-r_{2}, K\right]}{\arg \max }\left\{\tilde{\Pi}_{1}^{2,1}, \tilde{\Pi}_{1}^{2,2}, \tilde{\Pi}_{1}^{3,1}, \tilde{\Pi}_{1}^{3,2}\right\} \\
= & \begin{cases}\left(K-r_{2}, K\right], & \text { if } r_{2}<\frac{K}{2}, w \leq z_{1}-K, \\
{\left[\frac{K}{2}, K\right],} & \text { if } r_{2} \geq \frac{K}{2}, w \leq z_{1}-K, \\
0, & \text { if } w>z_{1}-K .\end{cases}
\end{aligned}
$$

$$
\begin{aligned}
\tilde{\Pi}_{1}^{23 *} & =\max _{r_{1} \in\left(K-r_{2}, K\right]}\left\{\tilde{\Pi}_{1}^{2,1}, \tilde{\Pi}_{1}^{2,2}, \tilde{\Pi}_{1}^{3,1}, \tilde{\Pi}_{1}^{3,2}\right\} \\
& = \begin{cases}\left(z_{1}-w-K\right)\left(K-r_{2}\right), & \text { if } r_{2}<\frac{K}{2}, w \leq z_{1}-K, \\
\left(z_{1}-w-K\right) \frac{K}{2}, & \text { if } r_{2} \geq \frac{K}{2}, w \leq z_{1}-K, \\
0, & \text { if } w>z_{1}-K .\end{cases}
\end{aligned}
$$

(i) Consider the case when $w \in\left(0, z_{1}-2 K\right]$. Since $w \leq z_{1}-2 K$, it follows that $\left(z_{1}-w-r_{2}\right) / 2>K-r_{2}$, from Equations (1) and (2), we have $\tilde{r}_{1}^{1 *} \in\left(K-r_{2}, K\right]$ and $\tilde{\Pi}_{1}^{1 *}=\left(z_{1}-w-K\right)\left(K-r_{2}\right)$. Furthermore, since $w \leq z_{1}-2 K<z_{1}-K$, from Equations (3) and (4), we have (a) if $r_{2}<K / 2$, then $\tilde{r}_{1}^{23 *} \in\left(K-r_{2}, K\right]$ with $\tilde{\Pi}_{1}^{23 *}=\left(z_{1}-w-K\right)\left(K-r_{2}\right)$; (b) if $r_{2} \geq K / 2$, then $r_{1}^{23 *} \in[K / 2, K]$ with $\tilde{\Pi}_{1}^{23 *}=\left(z_{1}-w-K\right) K / 2$. Note that $\left(z_{1}-w-K\right)\left(K-r_{2}\right) \leq\left(z_{1}-w-K\right) K / 2$ due to $r_{2} \geq K / 2$, thus Lemma 1 (i) is easily verified.

(ii) To prove the remaining results of Lemma 1, let us first establish some useful results in the following lemma.

LEMMA A1: Define $\tilde{\alpha}_{1}=z_{1}-w-\sqrt{2 K\left(z_{1}-w-K\right)}, \quad \beta=$ $z_{1}-w+\sqrt{2 K\left(z_{1}-w-K\right)}$, if $w \in\left(z_{1}-3 K / 2, z_{1}-K\right]$, then $\tilde{\alpha}_{1}$ and $\beta$ are real numbers with $K / 2<\tilde{\alpha}_{1}<2 K+w-z_{1} \leq K \leq \beta$.

PROOF: Given any $w \in\left(z_{1}-3 K / 2, z_{1}-K\right]$, we have $2 K+w-z_{1}-$ $K / 2=w-\left(z_{1}-3 K / 2\right)<0$, thus $2 K+w-z_{1}>K / 2$. To show $\tilde{\alpha}_{1}>K / 2$ is equivalent to show $\left(z_{1}-w-K / 2\right)-\sqrt{2 K\left(z_{1}-w-K\right)}>$ 0 , or $\left[2\left(z_{1}-w\right)-K\right]^{2}>8 K\left(z_{1}-w-K\right)$. We can derive that $\left[2\left(z_{1}-w\right)-3 K\right]^{2}>0$, and thus we have $\tilde{\alpha}_{1}>K / 2$. Similarly, to show $\tilde{\alpha}_{1}<2 K+w-z_{1}-K / 2$, which is equivalent to $2\left(z_{1}-w-K\right)-$ $\sqrt{2 K\left(z_{1}-w-K\right)}<0$, or $2\left(z_{1}-w-K\right)<\sqrt{2 K\left(z_{1}-w-K\right)}$, it suffices to show $\sqrt{2\left(z_{1}-w-K\right)}<\sqrt{K}$. As $z_{1}-w-3 K / 2>0$, then we have $\tilde{\alpha}_{1}<2 K+w-z_{1}$. Furthermore, to verify $\tilde{\alpha}_{1}<K$ is the equivalent to show $\tilde{\alpha}_{1}-K=z_{1}-w-K-\sqrt{2 K\left(z_{1}-w-K\right)}<0$, or $\sqrt{z_{1}-w-K}<\sqrt{2 K}$. As $z_{1}-3 K-w<z_{1}-2 K-w<0$, we have $\tilde{\alpha}_{1}<K$. Similarly, we have $\beta-K=z_{1}-w-K+\sqrt{2 K\left(z_{1}-w-K\right)} \geq 0$, thus $\beta \geq K$. Hence, Lemma $\mathrm{A} 1$ is proved.

LEMMA A2: Suppose $w \in\left(z_{1}-3 K / 2, z_{1}-2 K\right]$, then we have:

(a) if $r_{2} \leq \tilde{\alpha}_{1}$, then $\left(z_{1}-w-r_{2}\right)^{2} / 4 \geq\left(z_{1}-w-K\right) K / 2$,

(b) if $r_{2}>\tilde{\alpha}_{1}$, then $\left(z_{1}-w-r_{2}\right)^{2} / 4<\left(z_{1}-w-K\right) K / 2$.

PROOF: Through simple algebra, we obtain $\left(z_{1}-w-r_{2}\right)^{2} / 4-\left(z_{1}-\right.$ $w-K) K / 2=\frac{1}{4}\left(r_{2}-\tilde{\alpha}_{1}\right)\left(r_{2}-\beta\right)$, thus, the lemma follows from Lemma A1.

Now, we discuss the scenario as $w \in\left(z_{1}-2 K, z_{1}-3 K / 2\right]$. Consider three cases: (a) if $r_{2} \leq 2 K-z_{1}+w$, or equivalently $\left(z_{1}-w-r_{2}\right) / 2 \leq K-r_{2}$, then it follows that $r_{1}^{1 *}=\left(z_{1}-w-r_{2}\right) / 2$ and $\tilde{\Pi}_{1}^{1 *}=\left(z_{1}-w-r_{2}\right)^{2} / 4$. On the other hand, if $r_{2} \leq 2 K-z_{1}+w \leq K / 2$, then we have $r_{1}^{23 *} \in\left(K-r_{2}, K\right]$ and $\tilde{\Pi}_{1}^{23 *}=\left(z_{1}-w-K\right)\left(K-r_{2}\right)$. Comparing $\tilde{\Pi}_{1}^{1 *}$ with $\tilde{\Pi}_{1}^{23 *}$, we have $\tilde{\Pi}_{1}^{1 *}-\tilde{\Pi}_{1}^{23 *}=\left(r_{2}+z_{1}-w-2 K\right)^{2} \geq 0$. Hence, we can derive the results as follows: (a) if $r_{2} \leq 2 K-z_{1}+w$, then $r_{1}\left(r_{2}\right)=r_{1}^{1 *}=\left(z_{1}-w-r_{2}\right) / 2$ and $\tilde{\Pi}_{1}\left(r_{2}, w\right)=\tilde{\Pi}_{1}^{1 *}=\left(z_{1}-w-r_{2}\right)^{2} / 4$; (b) if $2 K-z_{1}+w<r_{2} \leq K / 2$, or equivalently $\left(z_{1}-w-r_{2}\right) / 2>K-r_{2}$, it follows that $r_{1}^{1 *}=r_{1}^{23 *} \in$ $\left(K-r_{2}, K\right]$ and $\tilde{\Pi}_{1}^{1 *}=\tilde{\Pi}_{1}^{23 *}=\left(z_{1}-w-K\right)\left(K-r_{2}\right)$; (c) if $r_{2}>K / 2$, then $\left(z_{1}-w-K\right)\left(K-r_{2}\right)<\left(z_{1}-w-K\right) K / 2$, and $r_{1}\left(r_{2}\right)=r_{1}^{23 *} \in[K / 2, K]$ with $\tilde{\Pi}_{1}\left(r_{2}, w\right)=\tilde{\Pi}_{1}^{23 *}=\left(z_{1}-w-K\right) K / 2$. Using the same procedure, we can verify Lemma 1(iii).

(iv) $w \in\left(z_{1}-K, z_{1}\right)$. As $w>z_{1}-K$, from Equation 4, we know that $\tilde{\Pi}_{1}^{23 *}=0$, so we only need to consider $\tilde{\Pi}_{1}^{1 *}$ from Equations (1) and (2). Consider two cases: (a) if $r_{2} \leq z_{1}-w$, then $0 \leq \frac{z_{1}-w-r_{2}}{2} \leq K-r_{2}$, and thus $r_{1}\left(r_{2}\right)=r_{1}^{1 *}=\left(z_{1}-w-r_{2}\right) / 2$, and $\tilde{\Pi}_{1}\left(r_{2}, w\right)=\tilde{\Pi}_{1}^{1 *}=\left(z_{1}-w-r_{2}\right)^{2} / 4$; (b) if $r_{2}>z_{1}-w$, then $\frac{z_{1}-w-r_{2}}{2}<0$, and thus $r_{1}\left(r_{2}\right)=r_{1}^{1 *}=0$ with $\tilde{\Pi}_{1}\left(r_{2}, w\right)=\tilde{\Pi}_{1}^{1 *}=0$. 


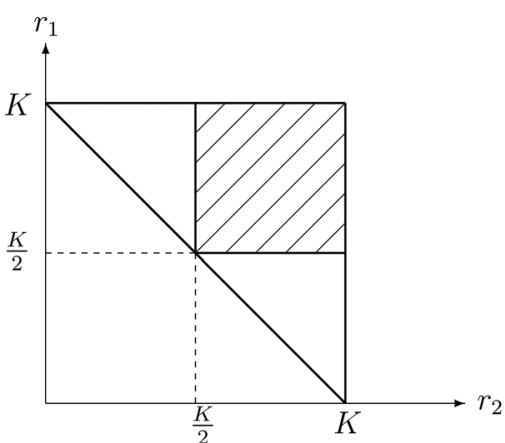

Figure $(\mathrm{A}-\mathrm{a}) w \in\left(0, z_{2}-2 K\right]$

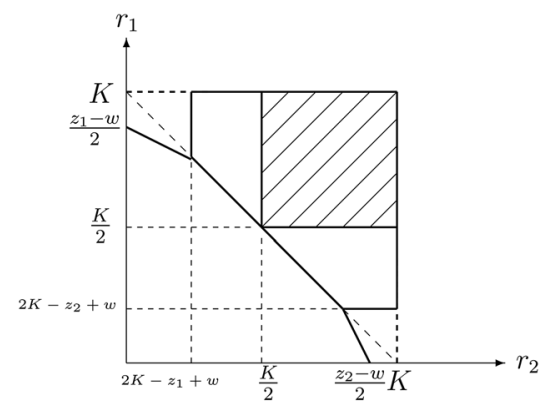

Figure (A-c) $w \in\left(z_{1}-2 K, z_{2}-3 K / 2\right]$

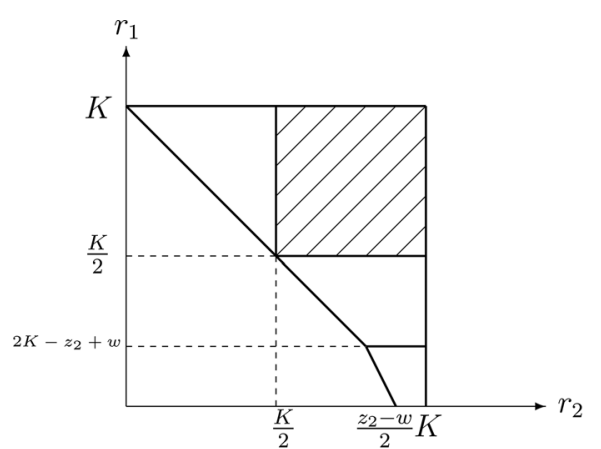

Figure $(\mathrm{A}-\mathrm{b}) w \in\left(z_{2}-2 K, z_{1}-2 K\right]$

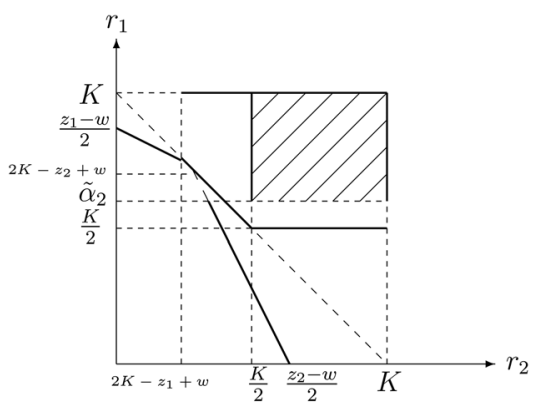

Figure (A-d) $w \in\left(z_{2}-3 K / 2, z_{1}-3 K / 2\right]$
PROOF OF THEOREM 1: To find Nash equilibrium outcome resulting from the ordering quantity game, we draw the response curves from Lemma 1 for both retailers. First, we compartmentalize the following feasible intervals with different capacity constraints. There are five possible scenarios: (I) if $z_{1}-z_{2}<K / 2$, i.e., $K>2\left(z_{1}-z_{2}\right)$, we have $z_{2}-2 K<z_{1}-2 K<$ $z_{2}-3 K / 2<z_{1}-3 K / 2<z_{2}-K<z_{1}-K<z_{2}<z_{1}$; (II) if $K / 2 \leq z_{1}-z_{2}<K$, that is, $\left.z_{1}-z_{2}<K \leq 2\left(z_{1}-z_{2}\right)\right]$, then we have $z_{2}-2 K \leq z_{2}-3 K / 2<z_{1}-2 K<z_{2}-K<z_{1}-3 K / 2 \leq z_{1}-K \leq$ $z_{2} \leq z_{1}$; (III) if $K \leq z_{1}-z_{2}<3 K / 2$, that is, $2\left(z_{1}-z_{2}\right) / 3<K \leq z_{1}-z_{2}$, then we have $z_{2}-2 K \leq z_{2}-3 K / 2 \leq z_{2}-K \leq z_{1}-2 K \leq$ $z_{1}-3 K / 2<z_{2} \leq z_{1}-K<z_{1}$; (IV) if $3 K / 2 \leq z_{1}-z_{2}<2 K$, that is, $\left(z_{1}-z_{2}\right) / 2<K \leq 2\left(z_{1}-z_{2}\right) / 3$, then we have $z_{2}-2 K<z_{2}-3 K / 2<$ $z_{2}-K<z_{1}-2 K<z_{2} \leq z_{1}-3 K / 2<z_{1}-K<z_{1}$; (V) if $z_{1}-z_{2} \geq 2 K$, i.e., $K \leq\left(z_{1}-z_{2}\right) / 2$, then we have $z_{2}-2 K<z_{2}-3 K / 2<z_{2}-K<z_{2} \leq$ $z_{1}-2 K<z_{1}-3 K / 2<z_{1}-K<z_{1}$. Next, we analyze Nash equilibrium in each scenario.

(I) $K \in\left(2\left(z_{1}-z_{2}\right),+\infty\right)$ :

(i) $w \in\left(0, z_{2}-2 K\right)$. In this case, See Fig. (A-a), it is clear that any point in $[K / 2, K] \times[K / 2, K]$ is a Nash equilibrium.

(ii) $w \in\left(z_{2}-2 K, z_{1}-2 K\right]$. Although it adds a new bound line $r_{1}=2 K-z_{2}+w$ as $r_{1} \leq 2 K-z_{2}+w$, and the response curve for retailer 2 is $r_{2}\left(r_{1}\right)=\left(z_{2}-w-r_{1}\right) / 2$, thus the equilibrium orders are the same as (i).

(iii) $w \in\left(z_{1}-2 K, z_{2}-3 K / 2\right]$. See Fig. (A-c), any point in $[K / 2, K] \times[K / 2, K]$ is in equilibrium.

(iv) $w \in\left(z_{2}-3 K / 2, z_{1}-3 K / 2\right]$. As showed in Fig. (Ad), any point in $\left[\tilde{\alpha}_{2}, K\right] \times[K / 2, K]$ is a Nash equilibrium. Next we determine whether the point $\left(\left(2 z_{1}-z_{2}-\right.\right.$ $\left.w) / 3,\left(2 z_{2}-z_{1}-w\right) / 3\right)$ is in equilibrium. It suffices to verify the conditions: (a) $\left(2 z_{1}-z_{2}-w\right) / 3 \leq \tilde{\alpha}_{2}$; (b) $\left(2 z_{2}-z_{1}-w\right) / 3 \leq 2 K-z_{1}+w$. Note from (a), it is equivalent to $w \leq \tilde{w}^{-}$or $\tilde{w}^{+} \leq w \leq 2 z_{2}-z_{1}$, where $\tilde{w}^{ \pm}=2 z_{2}-z_{1}-9 K / 4 \pm 3 \sqrt{K^{2}+8 K\left(z_{1}-z_{2}\right)} / 4$. Recall that $w \in\left(z_{2}-3 K / 2, z_{1}-3 K / 2\right]$, together with $\tilde{w}^{-}<z_{2}-3 K / 2$ and $z_{2}-3 K / 2<\tilde{w}^{+}<$ $z_{1}-3 K / 2<2 z_{2}-z_{1}$, simple algebra shows that condition (a) always holds if $w \in\left(\tilde{w}^{+}, z_{1}-3 K / 2\right]$. Conversely, for condition (b), it satisfies $w \geq\left(z_{1}+z_{2}-3 K\right) / 2$ if it holds. Note that $\left(z_{1}+z_{2}-3 K\right) / 2<\tilde{w}^{+}$, as a result, if $w \in\left(z_{2}-3 K / 2, \tilde{w}^{+}\right]$, then any point in $\left[\tilde{\alpha}_{2}, K\right] \times[K / 2, K]$ is a Nash equilibrium. Otherwise if $w \in\left(\tilde{w}^{+}, z_{1}-3 K / 2\right]$, then $\left(\left(2 z_{1}-z_{2}-w\right) / 3,\left(2 z_{2}-\right.\right.$ $\left.z_{1}-w\right) / 3$ ) is an equilibrium and any point in $\left[\tilde{\alpha}_{2}, K\right] \times$ $[K / 2, K]$ is also a Nash equilibrium. Moreover, when $w \in\left(\tilde{w}^{+}, z_{1}-3 K / 2\right]$, in this case, retailer 2's profit is $\tilde{\Pi}_{2}^{1}\left(\left(2 z_{2}-z_{1}-w\right) / 3, w\right)=\left(2 z_{2}-z_{1}-w\right)^{2} / 9$ if the Nash equilibrium is $\left(\left(2 z_{1}-z_{2}-w\right) / 3,\left(2 z_{2}-z_{1}-w\right) / 3\right)$, and $\tilde{\Pi}_{2}^{2}(K / 2, w)=\left(z_{2}-w-K\right) K / 2$ if the Nash equilibrium is any point in $\left[\tilde{\alpha}_{2}, K\right] \times[K / 2, K]$, it is easy to verify that $\tilde{\Pi}_{2}^{1}\left(\left(2 z_{1}-z_{2}-w\right) / 3, w\right) \geq \tilde{\Pi}_{2}^{2}(K / 2, w)$, thus the former equilibrium dominates the latter one by gaining more profits for retailer 2 . Similarly, we can prove that the equilibrium $\left(\left(2 z_{1}-z_{2}-w\right) / 3,\left(2 z_{2}-z_{1}-w\right) / 3\right)$ dominates the other equilibrium by generating more profits for retailer 1 . Hence, $\left(\left(2 z_{1}-z_{2}-w\right) / 3,\left(2 z_{2}-z_{1}-w\right) / 3\right)$ is a dominant strategy for both retailers.

(v) $w \in\left(z_{1}-3 K / 2, z_{2}-K\right]$. In this case (Fig. A-e), it implies that $K / 2 \leq\left(z_{1}-w\right) / 2 \leq K$ and $K / 2 \leq$ $\left(z_{2}-w\right) / 2 \leq K$. Furthermore, if $r_{2}=K / 2$, then $r_{1}(K / 2)=\left(z_{1}-w-K / 2\right) / 2$, it is easy to check that $r_{1}(K / 2) \leq K / 2$. Similarly, if $r_{1}=K / 2$, it is easy to check that $r_{2}(K / 2) \leq K / 2$, then there must exist a crossing point $\left(\left(2 z_{1}-z_{2}-w\right) / 3,\left(2 z_{2}-z_{1}-\right.\right.$ $w) / 3$ ) between the two response curves. Consequently, 


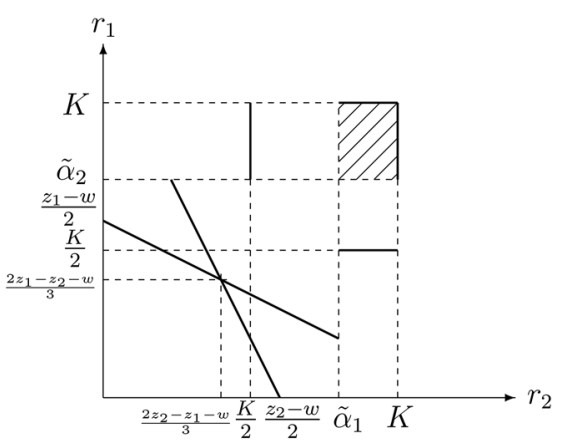

Figure (A-e) $w \in\left(z_{1}-3 K / 2, z_{2}-K\right]$

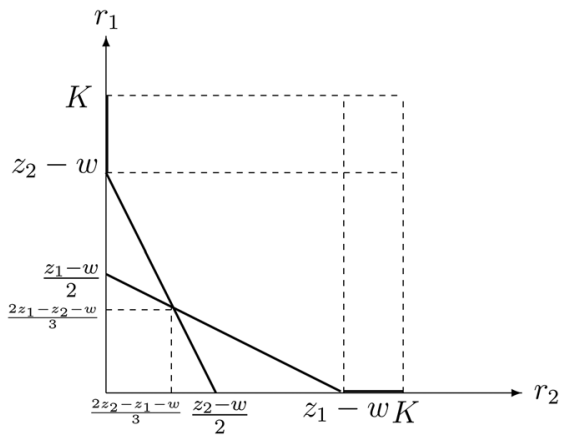

Figure $(\mathrm{A}-\mathrm{g}) w \in\left(z_{1}-K, 2 z_{2}-z_{1}\right]$

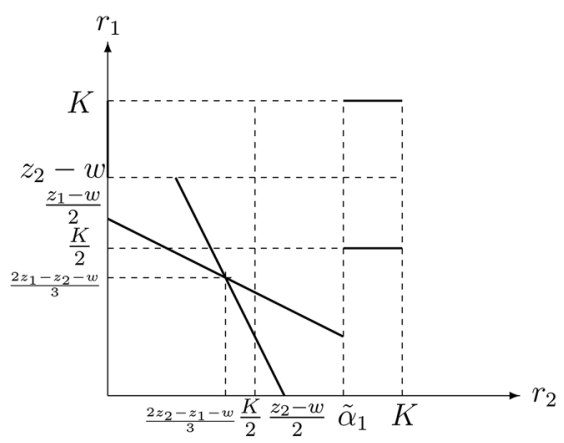

Figure (A-f) $w \in\left(z_{2}-K, z_{1}-K\right]$

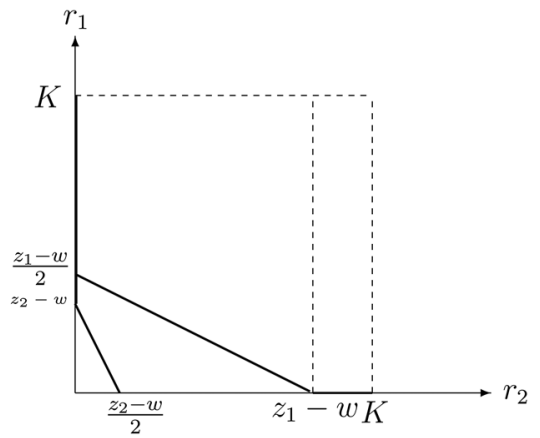

Figure (A-h) $w \in\left(2 z_{2}-z_{1}, z_{2}\right)$

(viii) $w \in\left(z_{2}, z_{1}\right)$. In this special case, the wholesale price is so high that retailer 2 would be driven out of the market. Therefore, see Fig. (A-i), the unique equilibrium is $\left(\left(z_{1}-w\right) / 2,0\right)$.

Moreover, we find that when $z_{1}-z_{2}<K \leq 2\left(z_{1}-z_{2}\right)$, the equilibrium orders are identical to the case as $K>2\left(z_{1}-z_{2}\right)$. Similarly, we use the same approach to analyze other scenarios when $K$ lies $\left(0, z_{1}-z_{2}\right]$. Therefore, we can summarize the results that show in Theorem 1.

PROPOSITION 1: Under uniform allocation mechanism with asymmetric retailers, the supplier's optimal wholesale price $\tilde{w}^{*}$ and the associated maximum profit denoted by $\tilde{\Pi}_{s}^{*}$, are as follows:

(I) $K \leq z_{1}-z_{2}$ :

(i) if $z_{2} \leq 3 z_{1} / 4$, when $K \leq z_{1} / 4$, the supplier's optimal wholesale price is $\tilde{w}^{*}=z_{1}-2 K$ and the maximum profit for the supplier is $\tilde{\Pi}_{s}^{*}=K\left(z_{1}-2 K\right)$; when $z_{1} / 4<K \leq z_{1}-z_{2}$, the supplier's optimal wholesale price is $\tilde{w}^{*}=z_{1} / 2$ and the maximum profit for the supplier is $\tilde{\Pi}_{s}^{*}=z_{1}^{2} / 8$

(ii) if $z_{2}>3 z_{1} / 4$, when $K \leq z_{1}-z_{2}$, then the supplier's optimal wholesale price is $\tilde{w}^{*}=z_{1}-2 K$ and the maximum profit for the supplier is $\tilde{\Pi}_{s}^{*}=K\left(z_{1}-2 K\right)$.

(II) $K>z_{1}-z_{2}$ :

(i) if $z_{2} \leq 5 z_{1} / 7$, when $K>z_{1}-z_{2}$, the supplier's optimal wholesale price is $\tilde{w}^{*}=\arg \max _{w \in\left\{\tilde{w}^{+}, z_{1} / 2\right\}} \tilde{\Pi}_{s}^{*}$ and the maximum profit for the supplier is $\tilde{\Pi}_{s}^{*}=$ $\max \left\{K \tilde{w}^{+}, z_{1}^{2} / 8\right\}$;

(ii) if $5 z_{1} / 7<z_{2} \leq 3 z_{1} / 4$, when $z_{1}-z_{2}<$ $K \leq \tilde{K}_{2}^{+}$, the supplier's optimal wholesale price is $\tilde{w}^{*}=\arg \max _{w \in\left\{\tilde{w}^{+}, z_{1} / 2\right\}} \tilde{\Pi}_{s}^{*}$ and the maximum profit

Figure $(\mathrm{A}-\mathrm{i}) w \in\left(z_{2}, z_{1}\right)$ 
for the supplier is $\tilde{\Pi}_{s}^{*}=\max \left\{K \tilde{w}^{+}, z_{1}^{2} / 8\right\}$. Otherwise, the supplier's optimal wholesale price is $\tilde{w}^{*}=$ $\arg \max _{w \in\left\{\tilde{w}^{+},\left(z_{1}+z_{2}\right) / 4, z_{1} / 2\right\}} \tilde{\Pi}_{s}^{*}$ and the maximum profit for the supplier is $\tilde{\Pi}_{s}^{*}=\max \left\{K \tilde{w}^{+},\left(z_{1}+z_{2}\right)^{2} / 24, z_{1}^{2} / 8\right\}$

(iii) if $z_{2}>3 z_{1} / 4$, when $z_{1}-z_{2}<K \leq \tilde{K}_{2}^{+}$, the supplier's optimal wholesale price is $\tilde{w}^{*}=$ $\arg \max _{w \in\left\{\tilde{w}^{+}, 2 z_{2}-z_{1}\right\}} \tilde{\Pi}_{s}^{*}$ and the maximum profit for the supplier is $\tilde{\Pi}_{s}^{*}=\max \left\{K \tilde{w}^{+},\left(2 z_{2}-z_{1}\right)\left(z_{1}-\right.\right.$ $\left.\left.z_{2}\right)\right\}$. Otherwise, the supplier's optimal wholesale price is $\tilde{w}^{*}=\arg \max _{w \in\left\{\tilde{w}^{+},\left(z_{1}+z_{2}\right) / 4,2 z_{2}-z_{1}\right\}} \tilde{\Pi}_{s}^{*}$ and the maximum profit for the supplier is $\tilde{\Pi}_{s}^{*}=$ $\max \left\{K \tilde{w}^{+},\left(z_{1}+z_{2}\right)^{2} / 24,\left(2 z_{2}-z_{1}\right)\left(z_{1}-z_{2}\right)\right\}$.

PROOF: Consider the following two cases.

Case (I): $K \leq z_{1}-z_{2}$ :

(i) $w \in\left(0, z_{1}-2 K\right]$. It is easy to show that $\tilde{w}^{*}=z_{1}-2 K$ with $\tilde{\Pi}_{s}^{1}=K\left(z_{1}-2 K\right)$

(ii) $w \in\left(z_{1}-2 K, z_{1}\right)$. (a) If $z_{1}-2 K<z_{1} / 2$, which is equivalent to $K>z_{1} / 4$, then $\tilde{w}^{*}=z_{1} / 2$ with $\tilde{\Pi}_{s}^{2}=z_{1}^{2} / 8$. (b) if $K \leq z_{1} / 4$, then $\tilde{w}^{*}=z_{1}-2 K$ with $\tilde{\Pi}_{s}^{2}=\tilde{\Pi}_{s}^{2}=K\left(z_{1}-2 K\right)$. Since $z_{1}^{2} / 8-K\left(z_{1}-2 K\right)=\left(8 K-z_{1}\right)^{2} / 8 \geq 0$, it follows that $z_{1}^{2} / 8 \geq K\left(z_{1}-2 K\right)$.

Case (II): $K>z_{1}-z_{2}$ :

(i) $w \in\left(0, \tilde{w}^{+}\right]$. It is easy to show that $\tilde{w}^{*}=\tilde{w}^{+}$with $\tilde{\Pi}_{s}^{3}=K \tilde{w}^{+}$;

(ii) $w \in\left(\tilde{w}^{+}, 2 z_{2}-z_{1}\right]$. (a) Consider the scenario where $2 z_{2}-$ $z_{1}>\left(z_{1}+z_{2}\right) / 4$, i.e., $z_{2}>5 z_{1} / 7$. If $\tilde{w}^{+}<\left(z_{1}+z_{2}\right) / 4$, which is equivalent to $K \geq \tilde{K}_{2}^{+}$, then $\tilde{w}^{*}=\left(z_{1}+z_{2}\right) / 4$ with $\tilde{\Pi}_{s}^{4}=\left(z_{1}+z_{2}\right)^{2} / 24$. If $\tilde{w}^{+} \geq\left(z_{1}+z_{2}\right) / 4$, then $\tilde{w}^{*}=\tilde{w}^{+}$with $\tilde{\Pi}_{s}^{4}=\left(z_{1}+z_{2}-\tilde{w}^{+}\right) \tilde{w}^{+} / 3$. (b) Consider the scenario where $2 z_{2}-z_{1} \leq\left(z_{1}+z_{2}\right) / 4$, i.e., $z_{2} \leq 5 z_{1} / 7$. It is easy to verify that $\tilde{w}^{*}=2 z_{2}-z_{1}$ with $\tilde{\Pi}_{s}^{4}=\left(2 z_{2}-z_{1}\right)\left(z_{1}-z_{2}\right)$.

(iii) $w \in\left(2 z_{2}-z_{1}, z_{1}\right)$. (a) If $2 z_{2}-z_{1} \leq z_{1} / 2$, i.e., $z_{2} \leq 3 z_{1} / 4$, then it follows that $\tilde{w}^{*}=z_{1} / 2$ with $\tilde{\Pi}_{s}^{5}=z_{1}^{2} / 8$. (b) If $2 z_{2}-z_{1}>z_{1} / 2$, that is, $z_{2}>3 z_{1} / 4$, then it follows that $\tilde{w}^{*}=2 z_{2}-z_{1}$ with $\tilde{\Pi}_{s}^{5}=\left(2 z_{2}-z_{1}\right)\left(z_{1}-z_{2}\right)$.

The proposition directly follows the above discussions.

LEMMA 2: Let $\hat{r}_{i}\left(r_{j}\right)$ be retailer $i$ 's best response order quantity given retailer $j$ 's order $(i, j=1,2$ and $i \neq j)$. Define $\hat{\alpha}_{i}=\left(K+z_{i}-w-\right.$ $\left.\sqrt{\left(z_{i}-w-K\right)\left(z_{i}-w+7 K\right)}\right) / 2$; then we have

(i) $w \in\left(0, z_{i}-2 K\right]$ : for any $r_{j}, \hat{r}_{i}\left(r_{j}\right)=K$, and $\hat{\Pi}_{i}\left(r_{j}, w\right)=$ $K^{2}\left(z_{i}-K-w\right) /\left(K+r_{j}\right)$.

(ii) $w \in\left(z_{i}-2 K, z_{i}-K\right.$ ]: if $r_{j} \leq \hat{\alpha}_{i}$, then $\hat{r}_{i}\left(r_{j}\right)=\left(z_{i}-w-r_{j}\right) / 2$ with $\hat{\Pi}_{i}\left(r_{j}, w\right)=\left(z_{i}-w-r_{j}\right)^{2} / 4$; if $r_{j}>\hat{\alpha}_{i}$, then $\hat{r}_{i}\left(r_{j}\right)=K$ with $\hat{\Pi}_{i}\left(r_{j}, w\right)=K^{2}\left(z_{i}-w-K\right) /\left(K+r_{j}\right)$.

(iii) $w \in\left(z_{i}-K, z_{i}\right)$ : if $r_{j} \leq z_{i}-w$, then $\hat{r}_{i}\left(r_{j}\right)=\left(z_{i}-w-r_{j}\right) / 2$ with $\hat{\Pi}_{i}\left(r_{j}, w\right)=\left(z_{i}-w-r_{j}\right)^{2} / 4$; if $r_{j}>z_{i}-w$, then $\hat{r}_{i}\left(r_{j}\right)=0$ with $\hat{\Pi}_{i}\left(r_{j}, w\right)=0$.

The proof of Lemma 2 is similar to the symmetric case (retailers have the same market power) of Chen et al. (2013), and thus, here we omit the details. Note that by involving asymmetric market power, each retailer's best response order size is not only affected by the wholesale price but also by the other retailer's order quantity. Take retailer 1 for example. We interpret Lemma 2 as follows. First, consider the case where the wholesale price is very low; that is, when $w \in\left(0, z_{i}-2 K\right]$. Lemma 2 (i) suggests that no matter what quantity the other retailer orders, retailer 1 will order $K$ to maximize her profit. This is because, when $w \leq z_{1}-2 K$, that is, $K \leq\left(z_{1}-w\right) / 2$, if the capacity level is relatively low, then retailer 1 would order as much as possible. Second, as Lemma 2(iii) states, when the wholesale price is very high, each retailer will not order much $(\leq K)$ : (a) retailer 1 may not order when $z_{1}-w-K<0$ under the condition that retailer 2 orders more than $z_{2}-w$; (b) if retailer 2's order size is no more than $z_{1}-w$, which means that the remaining capacity is ample for retailer 1 , then the best response for retailer 1 is the same as the case without capacity constraint. Third, consider the case where the wholesale price is in the intermediate range, that is, $w \in\left[z_{1}-2 K, z_{1}\right)$. Observe from the second part of Lemma 2(ii), if retailer 2 orders sufficiently high $\left(>\hat{\alpha}_{i}\right)$, then the remaining capacity is scarce for retailer 1 . As the profit margin for retailer 1 remains $z_{1}-w-K \geq 0$, it follows that her profit is increasing in her order size and thus ordering the maximum capacity $K$ is her best response. If retailer 2 orders no more than $\hat{\alpha}_{i}$, then the result is consistent with the first part of Lemma 2(iii).

PROPOSITION 2: Let $\left(\hat{r}_{1}^{*}, \hat{r}_{2}^{*}\right)$ be equilibrium orders under proportional mechanism. Define $\hat{w}^{-}=3 K+\frac{z_{1}+z_{2}}{2}-\frac{3}{2} \sqrt{8 K^{2}+\left(z_{1}-z_{2}\right)^{2}}$, then we have

(I) $K \leq z_{1}-z_{2}$ :

(i) if $w \in\left(0, z_{2}-K\right]$, then there is a unique Nash equilibrium $\left(\hat{r}_{1}^{*}, \hat{r}_{2}^{*}\right)=(K, K)$

(ii) if $w \in\left(z_{2}-K, z_{1}-2 K\right]$, then there is a unique Nash equilibrium $\left(\hat{r}_{1}^{*}, \hat{r}_{2}^{*}\right)=(K, 0)$;

(iii) if $w \in\left(z_{1}-2 K, z_{1}\right)$, then there is a unique Nash equilibrium $\left(\hat{r}_{1}^{*}, \hat{r}_{2}^{*}\right)=\left(\left(z_{1}-w\right) / 2,0\right)$.

(II) $K>z_{1}-z_{2}$ :

(i) if $w \in\left(0, \hat{w}^{-}\right]$, then there is a unique Nash equilibrium $\left(\hat{r}_{1}^{*}, \hat{r}_{2}^{*}\right)=(K, K)$

(ii) if $w \in\left(\hat{w}^{-}, z_{2}-K\right]$, then there exists two Nash equilibrium $(K, K)$ and $\left(\left(2 z_{1}-z_{2}-w\right) / 3,\left(2 z_{2}-z_{1}-w\right) / 3\right)$. Furthermore, the latter equilibrium dominates the former one by generating more profits for both retailers.

(iii) if $w \in\left(z_{2}-K, 2 z_{2}-z_{1}\right]$, then there is a unique Nash equilibrium $\left(\hat{r}_{1}^{*}, \hat{r}_{2}^{*}\right)=\left(\left(2 z_{1}-z_{2}-w\right) / 3,\left(2 z_{2}-z_{1}-\right.\right.$ $w) / 3$;

(iv) if $w \in\left(2 z_{2}-z_{1}, z_{1}\right)$, then there is a unique Nash equilibrium $\left(\hat{r}_{1}^{*}, \hat{r}_{2}^{*}\right)=\left(\hat{q}_{1}^{*}, \hat{q}_{2}^{*}\right)=\left(\left(z_{1}-w\right) / 2,0\right)$.

PROOF: Before the equilibrium analysis, from Lemma 2, we also obtain some critical values of $w$, that is, $z_{1}-2 K, z_{1}-K, z_{2}-2 K, z_{2}-K, z_{1}, z_{2}$. Using simple algebraic calculus, it follows that: (I) when $K>z_{1}-z_{2}$, we have $z_{2}-2 K<z_{1}-2 K \leq z_{2}-K<z_{1}-K<z_{2}<z_{1}$; (II) when $\left(z_{1}-z_{2}\right) / 2<K \leq z_{1}-z_{2}$, we have $z_{2}-2 K<z_{2}-K \leq z_{1}-2 K<$ $z_{2} \leq z_{1}-2 K<z_{2} \leq z_{1}-K \leq z_{1}$; and (III) when $K \leq\left(z_{1}-z_{2}\right) / 2$, we have $z_{2}-2 K \leq z_{2}-K<z_{1}-2 K \leq z_{2}<z_{1}-K \leq z_{1}$.

We first discuss the case when $K \in\left(z_{1}-z_{2},+\infty\right)$.

(i) $w \in\left(0, z_{2}-2 K\right] \cup\left(z_{2}-2 K, z_{1}-2 K\right]$. See the curves of response functions pictured in Fig. (B-a) and Fig. (B-b), it is easy to see that $(K, K)$ is the unique equilibrium.

(ii) $w \in\left(z_{1}-2 K, z_{2}-K\right]$. First let us introduce a critical value $\hat{w}^{-}$. From Lemma 1, the sufficient and necessary conditions for the ideal equilibrium orders $\left(\left(2 z_{1}-z_{2}-w\right) / 3,\left(2 z_{2}-z_{1}-w\right) / 3\right)$ are $\left(2 z_{1}-z_{2}-w\right) / 3 \leq \hat{\alpha}_{2}$ and $\left(2 z_{2}-z_{1}-w\right) / 3 \leq \hat{\alpha}_{1}$, which is equivalent to satisfy $\hat{w}^{-} \leq w \leq \hat{w}^{+}$, where $\hat{w}^{ \pm}=3 K+\frac{z_{1}+z_{2}}{2}-$ $\frac{3}{2} \sqrt{8 K^{2}+\left(z_{1}-z_{2}\right)^{2}}$. Due to $\hat{w}^{+}>z_{1}$ and the constraint $w<z_{1}$, therefore, $\left.\left(2 z_{1}-z_{2}-w\right) / 3,\left(2 z_{2}-z_{1}-w\right) / 3\right)$ is in equilibrium if and only if $w \geq \hat{w}^{-}$. Recall that $(K, K)$ is in equilibrium as $w \in\left(z_{1}-2 K, z_{2}-K\right]$, then we have the following results: (a) if 


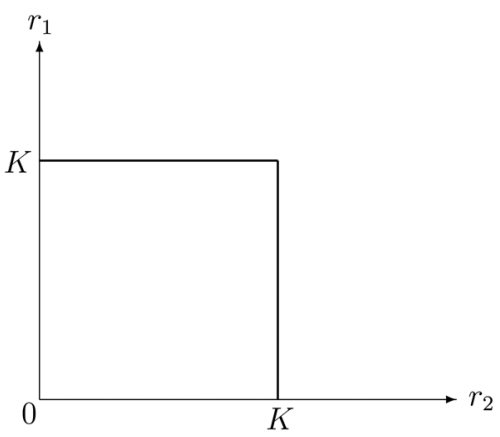

Figure (B-a) $0<w \leq z_{2}-2 K$

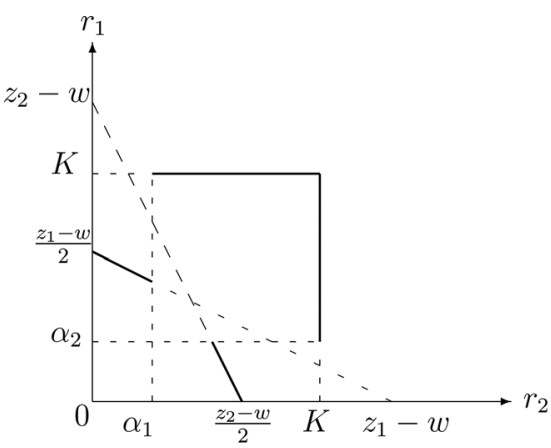

Figure (B-c) $z_{1}-2 K<w \leq \hat{w}^{-}$

$w \in\left(z_{1}-2 K, \hat{w}^{-}\right]$, there exists a unique Nash equilibrium $(K, K)$; (b) otherwise, $(K, K)$ and $\left(\left(2 z_{1}-z_{2}-w\right) / 3,\left(2 z_{2}-z_{1}-w\right) / 3\right)$ are both in equilibrium. Figure (B-c) and Fig. (B-d) illustrate the above results, respectively. Similar to the proof of Theorem 1, we can show that $\left(\left(2 z_{1}-z_{2}-w\right) / 3,\left(2 z_{2}-z_{1}-w\right) / 3\right)$ dominates $(K, K)$ by generating more profits for both retailers.

(iii) $w \in\left(z_{2}-K, z_{1}-K\right]$. In this scenario, if the orders $\left(\left(2 z_{1}-z_{2}-\right.\right.$ $\left.w) / 3,\left(2 z_{2}-z_{1}-w\right) / 3\right)$ are in equilibrium, it needs to satisfy two conditions: $\left(2 z_{1}-z_{2}-w\right) \leq\left(z_{1}-w\right) / 2$ and $\left(2 z_{2}-z_{1}-w\right) / 3 \leq$ $\left(z_{2}-w\right) / 2$. And the conditions are equivalent to $w \leq 2 z_{2}-z_{1}$. Together with the constraint $w \in\left(z_{2}-K, z_{1}-K\right]$, we obtain the results as follows: (a) when $z_{1}-z_{2}<K \leq 2\left(z_{1}-z_{2}\right)$, if $w \in\left(z_{2}-K, 2 z_{2}-z_{1}\right]$, there exist a unique Nash equilibrium $\left(\left(2 z_{1}-z_{2}-w\right) / 3,\left(2 z_{2}-z_{1}-w\right) / 3\right)$; otherwise, the unique equilibrium is $\left(\left(z_{1}-w\right) / 2,0\right)$; (b) when $K>2\left(z_{1}-z_{2}\right)$, $z_{1}-K<2 z_{1}-z_{2}$, then there exists a unique Nash equilibrium $\left(\left(2 z_{1}-z_{2}-w\right) / 3,\left(2 z_{2}-z_{1}-w\right) / 3\right)$. See Fig. (B-e) and Fig. (B-f), respectively. Specifically, the Figure of (b) is the same as the case with (a) when $w \in\left(z_{2}-K, 2 z_{2}-z_{1}\right]$.

(iv) $w \in\left(z_{1}-K, z_{2}\right]$. As pictured in Fig. (B-g) and (B-h), we have: (a) when $z_{1}-z_{2}<K \leq 2\left(z_{1}-z_{2}\right)$, there exists a unique Nash equilibrium $\left(\left(z_{1}-w\right) / 2,0\right)$; (b) when $K>2\left(z_{1}-z_{2}\right)$, if $w \in\left(z_{1}-K, 2 z_{2}-z_{1}\right]$, then there exists a unique Nash equilibrium $\left(\left(2 z_{1}-z_{2}-w\right) / 3,\left(2 z_{2}-z_{1}-w\right) / 3\right)$, otherwise, the unique equilibrium is $\left(\left(z_{1}-w\right) / 2,0\right)$. Specifically, the figure of scenario (a) is the same as the case (b) when $w \in\left(2 z_{2}-z_{1}, z_{2}\right)$.

(v) $w \in\left(z_{2}, z_{1}\right)$. It is the same as Fig. (A-i), in which the low-type retailer is driven out of the supply chain, then there is a unique Nash equilibrium $\left(\left(z_{1}-w\right) / 2,0\right)$.

Based on the above analysis, we can obtain the equilibrium outcome when $K>z_{1}-z_{2}$ in Theorem 2. Similarly, the remaining results follow from the

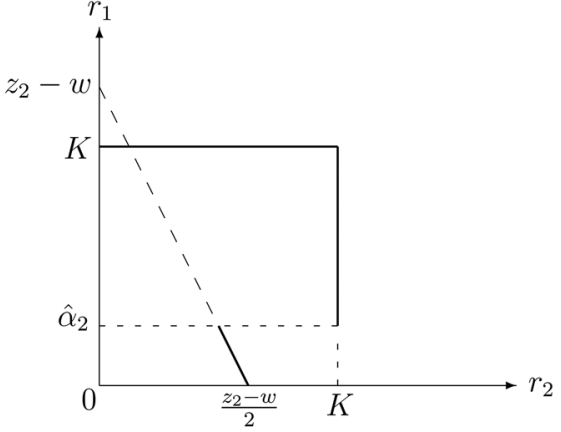

Figure (B-b) $z_{2}-2 K<w \leq z_{1}-2 K$

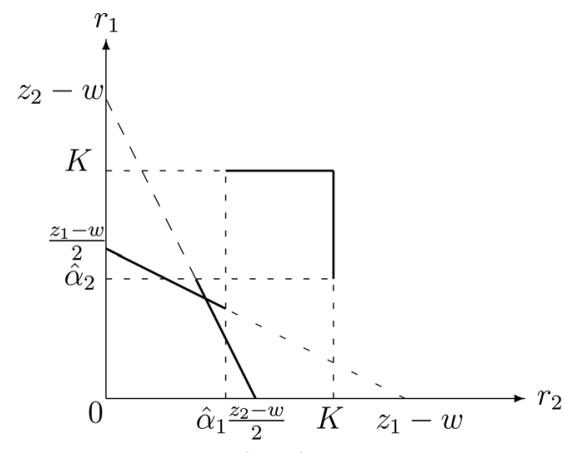

Figure (B-d) $\hat{w}^{-}<w<z_{2}-K$

same approach. As the step is similar to the case we have analysed, here we omit the details.

We interpret Proposition 2 in conjunction with the impact of wholesale price $w$ on orders in equilibrium, for any given capacity level satisfying $0<K \leq z_{1}-z_{2}$. When the wholesale price is very low, it is easy to check that the profit margin for each retailer is $z_{i}-w-K \geq 0,(i=1,2)$. Hence, both retailers order as much as possible (by ordering $K$ as allowed). Therefore, there exists a unique Nash equilibrium orders $(K, K)$. Conversely, when the wholesale price is sufficiently high, retailer 2 may be driven out of the game due to her lower market power; consequently, the equilibrium order quantity is always zero for retailer 2 in this case.

If capacity exceeds $z_{1}-z_{2}$, then we drive the equilibrium orders in four scenarios. First, consider the scenario with $w \in\left(0, \hat{w}^{-}\right]$. As $2 z_{2}-z_{1}-3 K / 2<$ $\left(z_{1}+z_{2}-3 K\right) / 2 \leq \hat{w}^{-}$, it follows that if $q_{1}^{*}+q_{2}^{*}<K \leq K\left(\hat{w}^{-}\right)$, where $K\left(\hat{w}^{-}\right)=\left(z_{1}+z_{2}-2 w+\sqrt{-7 z_{1}^{2}+22 z_{1} z_{2}-7 z_{2}^{2}-8 w\left(z_{1}+z_{2}-w\right)}\right) / 6$, then gaming effect will occur, that is, both retailers will strategically inflate their orders from $q_{i}^{*}$ to $K$. As a result, unique equilibrium orders $(K, K)$ exist when the wholesale price is relatively low. Second, when $w \in\left(\hat{w}, 2 z_{2}-z_{1}\right]$, there exist two equilibria $(K, K)$ and $\left(\left(2 z_{1}-z_{2}-w\right) / 3,\left(2 z_{2}-z_{1}-w\right) / 3\right)$. It is easy to verify that when substituting the resulted allocations into each retailer's profit function, the latter equilibrium dominates the former by generating higher profits for both retailers. Third, we consider $w \in\left(z_{2}-K, 2 z_{2}-z_{1}\right]$. In this case, the capacity is regarded as ample by both retailers, which order as in the case without a capacity limit. Fourth, we have the scenario $w \in\left(2 z_{2}-z_{1}, z_{1}\right)$. In this case, retailer 2 would be driven out of the market, which results in both equilibrium orders and allocations $\left(\left(z_{1}-w\right) / 2,0\right)$.

From Proposition 2, we have the following observation. When $0<K \leq$ $z_{1}-z_{2}$, as $w$ increases, the total equilibrium order quantities in the three scenarios of $w$ are (i) $2 K$, (ii) $K$, and (iii) $\frac{z_{1}-w}{2}$, respectively. Simple algebra shows that $2 K>K \geq \frac{z_{1}-w}{2}$. This is consistent with the intuition that total 


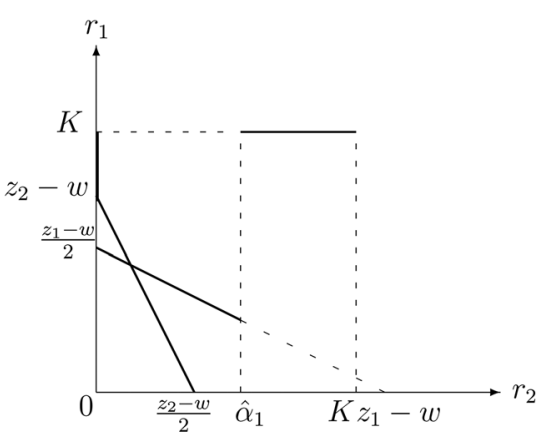

Figure (B-e) $z_{2}-K<w \leq 2 z_{2}-z_{1}$

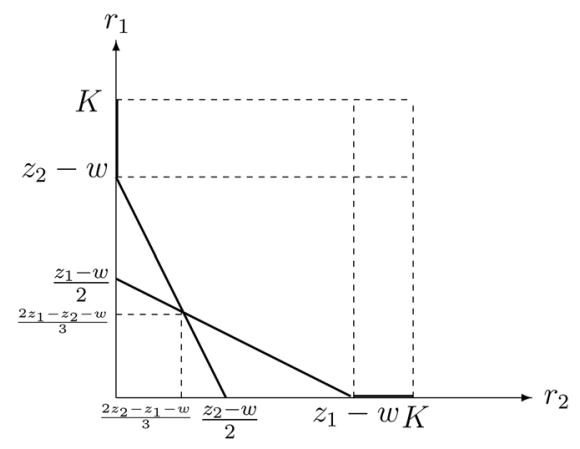

Figure (B-g) $w \in\left(z_{1}-K, 2 z_{2}-z_{1}\right]$

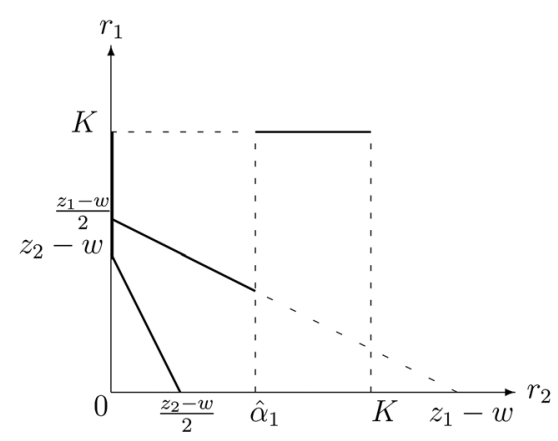

Figure (B-f) $2 z_{2}-z_{1}<w \leq z_{1}-K$

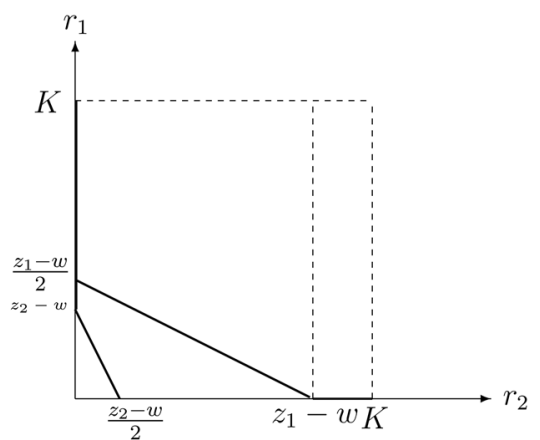

Figure (B-h) $w \in\left(2 z_{2}-z_{1}, z_{2}\right]$

(iv) if $w \in\left(z_{2}-K, 2 z_{2}-z_{1}\right]$, then there is a unique Nash equilibrium $\left(\check{r}_{1}^{*}, \check{r}_{2}^{*}\right)=\left(\left(2 z_{1}-z_{2}-w\right) / 3,\left(2 z_{2}-z_{1}-\right.\right.$ $w) / 3$;

(v) if $w \in\left(2 z_{2}-z_{1}, z_{1}\right)$, then there is a unique Nash equilibrium $\left(\check{r}_{1}^{*}, \check{r}_{2}^{*}\right)=\left(\left(z_{1}-w\right) / 2,0\right)$.

Second, suppose order priority is given to the low-type retailer (retailer 2). With a slight abuse of notation, let $\left(\check{r}_{1}^{*}, \breve{r}_{2}^{*}\right)$ be equilibrium orders under lexicographic allocation, and define $\check{\alpha}_{2}=z_{2}-w-\sqrt{4 K\left(z_{2}-w-K\right)}$, $\check{w}_{2}^{+}=2 z_{2}-z_{1}-\frac{9}{2} K+\frac{3}{2} \sqrt{5 K^{2}+4 K\left(z_{1}-z_{2}\right)}$. We have

PROPOSITION 3: First, suppose order priority is given to the hightype retailer (retailer 1). Let $\left(\check{r}_{1}^{*}, \breve{r}_{2}^{*}\right)$ be equilibrium orders under lexicographic allocation. Define $\check{\alpha}_{1}=z_{1}-w-\sqrt{4 K\left(z_{1}-w-K\right)}, \check{w}_{1}^{+}=$ $2 z_{1}-z_{2}-\frac{9}{2} K+\frac{3}{2} \sqrt{5 K^{2}-4 K\left(z_{1}-z_{2}\right)}$. We have

(I) $K \leq z_{1}-z_{2}$ :

(i) if $w \in\left(0, z_{2}-K\right]$, then any point in $\{K\} \times[0, K]$ is a Nash equilibrium;

(ii) if $w \in\left(z_{2}-K, z_{1}-2 K\right]$, then there is a unique Nash equilibrium $\left(\check{r}_{1}^{*}, \breve{r}_{2}^{*}\right)=(K, 0)$;

(iii) if $w \in\left(z_{1}-2 K, z_{1}\right)$, then there is a unique Nash equilibrium $\left(\check{r}_{1}^{*}, \check{r}_{2}^{*}\right)=\left(\left(z_{1}-w\right) / 2,0\right)$.

(II) $K>z_{1}-z_{2}$ :

(i) if $w \in\left(0, z_{1}-2 K\right]$, then any point in $\{K\} \times[0, K]$ is a Nash equilibrium;

(ii) if $w \in\left(z_{1}-2 K, \check{w}_{1}^{+}\right]$, then any point in $\{K\} \times\left[\check{\alpha}_{1}, K\right]$ is a Nash equilibrium;

(iii) if $w \in\left(\check{w}_{1}^{+}, z_{2}-K\right]$, then $\left(\left(2 z_{1}-z_{2}-w\right) / 3,\left(2 z_{2}-\right.\right.$ $\left.\left.z_{1}-w\right) / 3\right)$ is a Nash equilibrium and any point in $\{K\} \times\left[\check{\alpha}_{1}, K\right]$ is also a Nash equilibrium. Further, the former equilibrium dominates the latter by generating more profits for both retailers.
(I) $K \leq z_{1}-z_{2}$ :

(i) if $w \in\left(0, z_{2}-K\right]$, then any point in $[0, K] \times\{K\}$ is a Nash equilibrium;

(ii) if $w \in\left(z_{2}-K, z_{1}-2 K\right]$, then there is a unique Nash equilibrium $\left(\check{r}_{1}^{*}, \check{r}_{2}^{*}\right)=(K, 0)$;

(iii) if $w \in\left(z_{1}-2 K, z_{1}\right)$, then there is a unique Nash equilibrium $\left(\check{r}_{1}^{*}, \check{r}_{2}^{*}\right)=\left(\left(z_{1}-w\right) / 2,0\right)$.

(II) $K>z_{1}-z_{2}$ :

(i) if $w \in\left(0, z_{1}-2 K\right]$, then any point in $[0, K] \times\{K\}$ is a Nash equilibrium;

(ii) if $w \in\left(z_{1}-2 K, \check{w}_{2}^{+}\right]$, then any point in $\left[\check{\alpha}_{2}, K\right] \times\{K\}$ is a Nash equilibrium;

(iii) if $w \in\left(\check{w}_{2}^{+}, z_{2}-K\right]$, then $\left(\left(2 z_{1}-z_{2}-w\right) / 3,\left(2 z_{2}-\right.\right.$ $\left.\left.z_{1}-w\right) / 3\right)$ is a Nash equilibrium and any point in $\left[\check{\alpha}_{2}, K\right] \times\{K\}$ is also a Nash equilibrium. Further, the former equilibrium dominates the latter by generating more profits for both retailers.

(iv) if $w \in\left(z_{2}-K, 2 z_{2}-z_{1}\right]$, then there is a unique Nash equilibrium $\left(\check{r}_{1}^{*}, \check{r}_{2}^{*}\right)=\left(\left(2 z_{1}-z_{2}-w\right) / 3,\left(2 z_{2}-z_{1}-\right.\right.$ $w) / 3$; 
(v) if $w \in\left(2 z_{2}-z_{1}, z_{1}\right)$, then there is a unique Nash equilibrium $\left(\check{r}_{1}^{*}, \breve{r}_{2}^{*}\right)=\left(\left(z_{1}-w\right) / 2,0\right)$.

The proof of Proposition 3 is similar to that of Theorems 1 and 2, and here we omit the details. Proposition 3 is intuitive. Observe that for any given capacity level, the retailer with order priority is allowed to obtain the entire capacity if the wholesale price is very low. Interestingly, when the capacity level is relatively high, gaming effect also occurs. Note that when $K>z_{1}-$ $z_{2}$, if $w \in\left(\left(z_{i}+z_{j}-3 K\right) / 2, \breve{w}_{i}^{+}\right]$, satisfying $q_{1}^{*}+q_{2}^{*}<K \leq K\left(\check{w}_{i}^{+}\right)$, where $K\left(\check{w}_{i}^{+}\right)=\frac{z_{i}-w}{2}+\frac{1}{6} \sqrt{-7 z_{i}^{2}-2 z_{i} \check{w}_{i}+5 \check{w}_{i}^{2}+16 z_{i} z_{j}-4 z_{j}^{2}-8 z_{j} \check{w}}$, then retailer $i$ with order priority will order $K$, which is more than her ideal order size $r_{i}^{*}$. Further, if the wholesale price lies in the interval $\left(\check{w}_{i}^{*}, 2 z_{2}-z_{1}\right)$, then the retailers will order their ideal orders $\left(r_{1}^{*}, r_{2}^{*}\right)$ and lexicographic allocation is truth-inducing. However, if the wholesale price is sufficiently high, then the low-type retailer will order nothing whether or not she has order priority.

PROOF OF THEOREM 2: The theorem follows the discussions preceding the theorem in Section 5.

\section{Numerical Examples with Two Competing Retailers}

Figure 2 graphically illustrates the above finding for symmetric retailers. It is evident that as the capacity $K$ increases, the wholesale price in each allocation mechanism initially follows a descending trend and then remains unchanged when capacity becomes sufficiently large. Further, the supplier's profit under proportional allocation is no less than that under uniform allocation, but is no more than that under lexicographic allocation. We set forth the following interpretations for these results. As the supplier's capacity increases, to maximize his profit, it is beneficial for the supplier to reduce the wholesale price so as to entice the retailers to order more. But when the capacity is sufficiently large to meet all the retailer demand, both the total order quantity and the supplier's profit will stay at a constant level.

From Tables 1-3, our numerical studies are based on the following data: (I) when $\frac{z_{1}}{2}<z_{2} \leq \frac{5 z_{1}}{7}$, we have $\left(z_{1}, z_{2}\right)=(100,60)$ or $(100,65)$; (II) when $\frac{5 z_{1}}{7}<z_{2} \leq \frac{3 z_{1}}{4}$, we have $\left(z_{1}, z_{2}\right)=(100,72)$ or $(100,74)$; (III) when $\frac{3 z_{1}}{4}<z_{1}<z_{2}$, we have $\left(z_{1}, z_{2}\right)=(100,80)$ or $(100,90)$. Results are depicted in Figures 3-8.

Note that as $K$ increases, the supplier has the potential to sell more in the sense that he could obtain more profits unless the total ordering quantity by the two retailers is not increasing any more. The supplier may change the wholesale price strategically with capacity, as illustrated in Figures 3-8.

From Figures 3 and 4, it is evident that lexicographic allocation performs no differently than the other two allocations. Technically, this is because, although $K \check{w}_{1}^{+}>K \check{w}_{2}^{+}>K \hat{w}^{-}>K \tilde{w}^{+}$, the maximum of the four values is less than $z^{2} / 8$ for our numerical examples. Therefore, the supplier is indifferent among the three allocation mechanisms if the retailers' market powers are significantly different. However, as the low-type retailer's market power becomes closer to the high-type retailer's power, we observe from Figs. 5-8 that the supplier's profit obtained from lexicographic allocation is much higher than from uniform or proportional allocations, especially when order priority is given to the high-type retailer. This demonstrates that the advantage of superior mechanism in each comparison becomes more evident when the competition between the two retailers is more intense.

PROOF OF THEOREM 3: The proof is a similar to that of Theorem 5 and is omitted for preciseness.

PROOF OF THEOREM 4: First, we derive the values of $K_{u}^{*}, K_{p}^{*}, K_{l^{*}}^{*}$. Let $\tilde{q}_{i}^{\max }, \hat{q}_{i}^{\max }, \check{q}_{i}^{\max }$ be retailer $i$ 's largest received allocations under uniform, proportional and lexicographic (with priority sequence $l^{*}$ ) allocations given $r_{-i}=q_{-i}^{*}$, respectively. Specifically, we have

$$
\begin{aligned}
\tilde{q}_{i}^{\max } & =\frac{1}{\tilde{n}_{i}^{\prime}+1}\left(K-\sum_{j=\tilde{n}_{i}^{\prime}+1, \ldots, n ; j \neq i} q_{j}^{*}\right), \\
\hat{q}_{i}^{\max } & =\frac{K}{K+Q_{-i}^{*}} K, \\
\check{q}_{i}^{\max } & =K-\left(q_{1}^{*}+\cdots+q_{i-1}^{*}\right),
\end{aligned}
$$

where $\tilde{n}_{i}^{\prime}$ is defined as the largest integer less than $i$ such that $q_{\tilde{n}_{i}^{\prime}}^{*} \geq$ $\frac{1}{\hat{n}^{\prime}{ }_{i}+1}\left(K-\sum_{j=\tilde{n}_{i}^{\prime}+1, \cdots, n ; j \neq i} q_{j}^{*}\right)$. To ensure a positive marginal profit for retailer $i$ when the capacity is fully utilized, we assume that $z_{i}-w-K>0$, and thus for any retailer, an upper bound with $K<z_{n}-w$ is necessary for the assumption $z_{1}>z_{2}>\cdots>z_{n}$. Let $\Pi_{i}(x, y)$ be retailer $i$ 's profit with retailer $i$ 's allocation $x$ and the other retailers' total allocation $y$. Under uniform allocation, it is obvious that if there exists any retailer $i$ such that $\Pi_{i}\left(\tilde{q}_{i}^{\max }, K-\tilde{q}_{i}^{\max }\right) \geq \Pi_{i}\left(q_{i}^{*}, Q_{-i}^{*}\right)$, then the supplier's total allocated capacity is $K$. Otherwise, each retailer obtains her ideal allocation value. For notational convenience, let $\delta=\sum_{j=\tilde{n}_{i}^{\prime}+1, \ldots, n ; j \neq i} q_{j}^{*}$. We next obtain the threshold $K_{u}^{i *}$ for retailer $i$ as follows. Noting that $Q^{*}=\left(\Sigma_{j=1}^{n} z_{j}-n w\right) /(n+1)$ and $q_{i}^{*}=\left((n+1) z_{i}-\Sigma_{j=1}^{n} z_{j}-w\right) /(n+1)$ for their definitions, we immediately have the following equation,

$$
Q^{*}=z_{i}-w-q_{i}^{*}
$$

With Eq. (8), we have that $\Pi_{i}\left(\tilde{q}_{i}^{\max }, K-\tilde{q}_{i}^{\max }\right) \geq \Pi_{i}\left(q_{i}^{*}, Q_{-i}^{*}\right)$, which is equivalent to $\frac{z_{i}-w-K}{\tilde{n}_{i}^{\prime}+1}(K-\delta) \geq\left(z_{i}-w-Q^{*}\right) q_{i}^{*}$. Therefore, we have

$$
K^{2}-\left(z_{i}-w+\delta\right) K+\left(z_{i}-w\right) \delta+\left(\tilde{n}_{i}^{\prime}+1\right) q_{i}^{* 2} \leq 0 .
$$

Solving Eq. (9), we have

$$
\begin{aligned}
& \frac{z_{i}-w+\delta-\sqrt{\left[z_{i}-w+\delta\right]^{2}-4\left[\left(z_{i}-w\right) \delta+\left(\tilde{n}_{i}^{\prime}+1\right) q_{i}^{* 2}\right]}}{2} \leq K \\
& \quad \leq \frac{z_{i}-w+\delta+\sqrt{\left[z_{i}-w+\delta\right]^{2}-4\left[\left(z_{i}-w\right) \delta+\left(\tilde{n}_{i}^{\prime}+1\right) q_{i}^{* 2}\right]}}{2} .
\end{aligned}
$$

Note that $\frac{z_{i}-w+\delta-\sqrt{\left[z_{i}-w+\delta\right]^{2}-4\left[\left(z_{i}-w\right) \delta+\left(\tilde{n}_{i}^{\prime}+1\right) q_{i}^{* 2}\right]}}{2} \leq Q^{*} \leq$ $\frac{z_{i}-w+\delta+\sqrt{\left[z_{i}-w+\delta\right]^{2}-4\left[\left(z_{i}-w\right) \delta+\left(\tilde{n}_{i}^{\prime}+1\right) q_{i}^{* 2}\right]}}{2}$. Now we focus on the case with $K>Q^{*}$ in the theorem. We can obtain the threshold of retailer $i: K_{u}^{i *}=\frac{z_{i}-w+\delta+\sqrt{\left[z_{i}-w+\delta\right]^{2}-4\left[\left(z_{i}-w\right) \delta+\left(\tilde{n}_{i}^{\prime}+1\right) q_{i}^{* 2}\right]}}{2}$ and $K_{u}^{*}=$ $\max \left\{K_{u}^{1 *}, K_{u}^{2 *}, \ldots, K_{u}^{n *}\right\}$. Similar to the analysis in Fig. 1, gaming effect occurs when $K \in\left[Q^{*}, K_{u}^{*}\right]$.

Furthermore, we can use the same method to derive $K_{l^{*}}^{*}=$ $\max \left\{K_{l^{*}}^{1 *}, K_{l^{*}}^{2 *}, \ldots, K_{l^{*}}^{n *}\right\}, \quad K_{l^{*}}^{i *} \quad$ where $=$ $\underline{z_{i}-w+\sum_{j=1, \cdots, i-1} q_{j}^{*}+\sqrt{\left[z_{i}-w+\sum_{j=1, \cdots, i-1} q_{j}^{*}\right]^{2}-4 q_{i}^{* 2}}}$. Next, we need to obtain the value of $K_{p}^{*}$. We know that $\Pi_{i}\left(\hat{q}_{i}^{\max }, K-\hat{q}_{i}^{\max }\right)>\Pi_{i}\left(q_{i}^{*}, Q_{-i}^{*}\right)$, which is equivalent to $\left(z_{i}-w-K\right) \frac{K^{2}}{K+Q_{-i}^{*}}>q_{i}^{* 2}$. Then, we have that $-K^{3}+\left(z_{i}-w\right) K^{2}-q_{i}^{* 2} K-q_{i}^{* 2} Q_{-i}^{*}>0$. Let $f(K)=-K^{3}+\left(z_{i}-\right.$ w) $K^{2}-q_{i}^{* 2} K-q_{i}^{* 2} Q_{-i}^{*}$. It is obvious that function $f(K)$ is monotone and decreasing when $K \in\left[Q^{*}, z_{i}-w\right)$. From Eq. (8), we have

$$
\begin{aligned}
f\left(Q^{*}\right) & =-Q^{* 3}+\left(z_{i}-w\right) Q^{* 2}-q_{i}^{* 2} Q^{*}-q_{i}^{* 2} Q_{-i}^{*} \\
& =-Q^{* 3}+\left(Q^{*}+q_{i}^{*}\right) Q^{* 2}-q_{i}^{* 2} Q^{*}-q_{i}^{* 2}\left(Q^{*}-q_{i}^{*}\right) \\
& =q_{i}^{*}\left(Q^{*}-q_{i}^{*}\right)^{2}>0,
\end{aligned}
$$

Naval Research Logistics DOI 10.1002/nav 

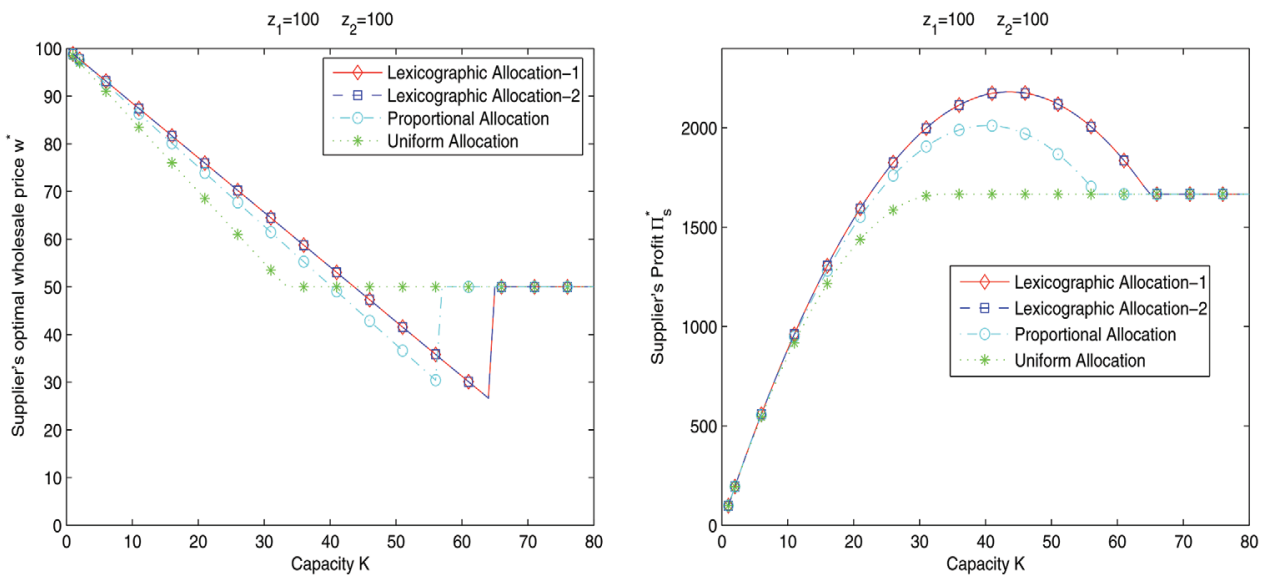

Figure 2. Comparison of Supplier's Pricing Decisions with Symmetric Retailers. [Color figure can be viewed at wileyonlinelibrary.com]
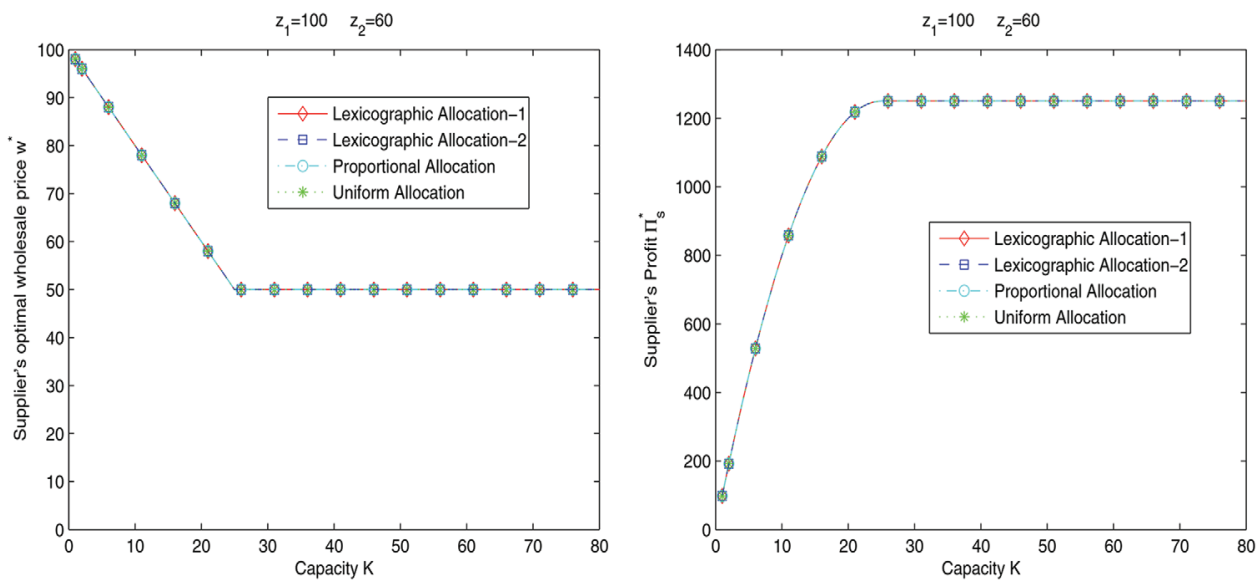

Figure 3. Comparison of Supplier's Pricing Decisions with Asymmetric Retailers. [Color figure can be viewed at wileyonlinelibrary.com]
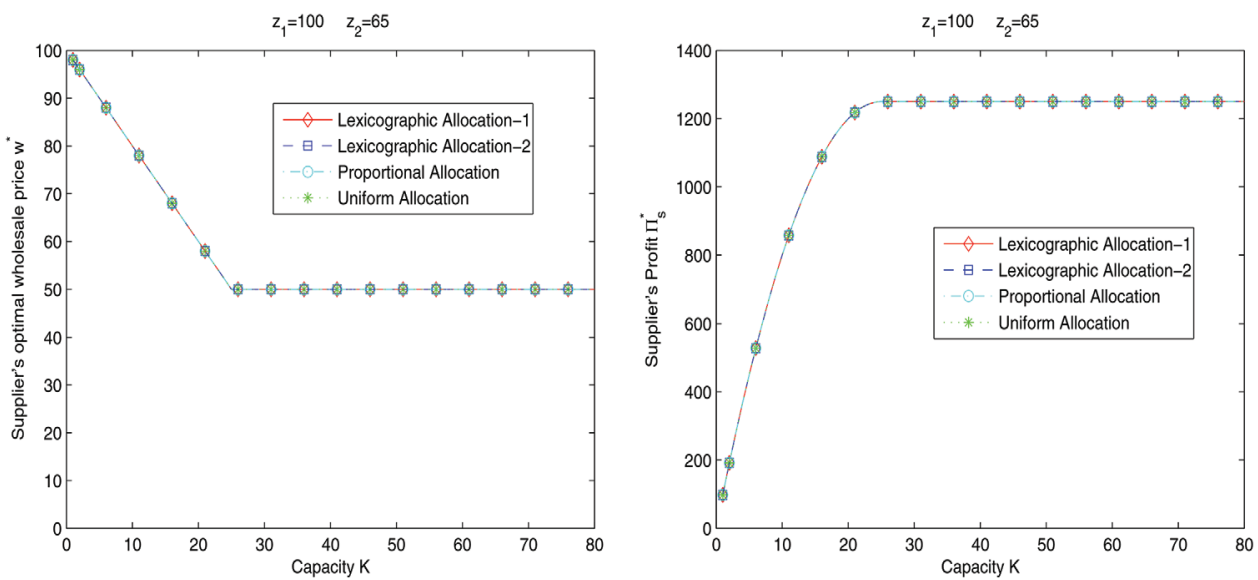

Figure 4. Comparison of Supplier's Pricing Decisions with Asymmetric Retailers. [Color figure can be viewed at wileyonlinelibrary.com] 

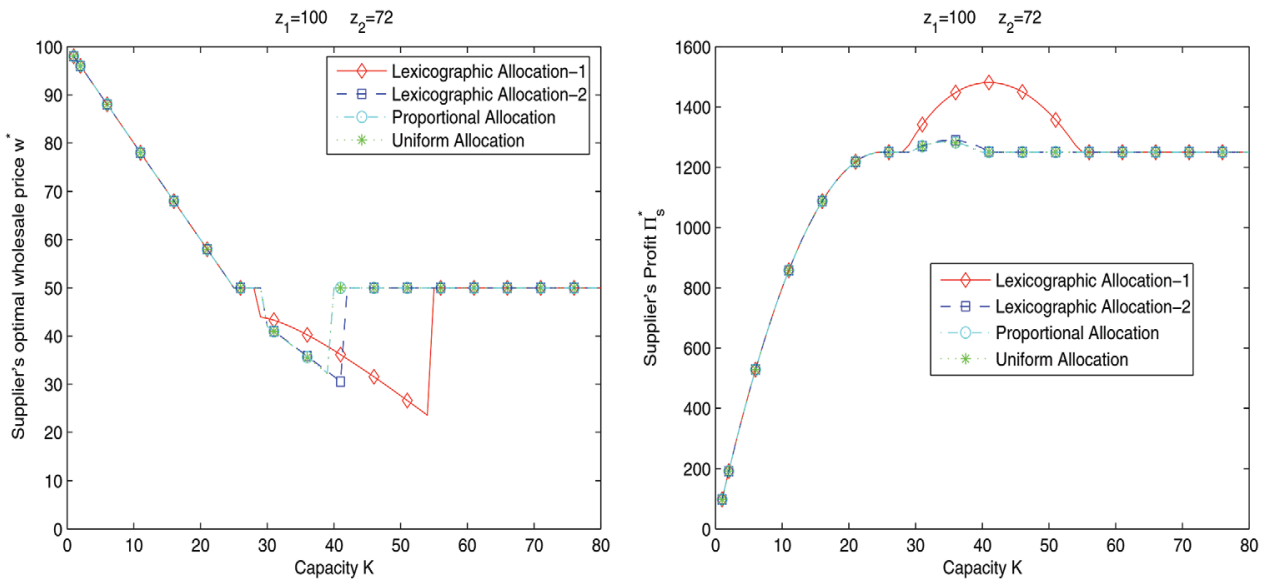

Figure 5. Comparison of Supplier's Pricing Decisions with Asymmetric Retailers. [Color figure can be viewed at wileyonlinelibrary.com]
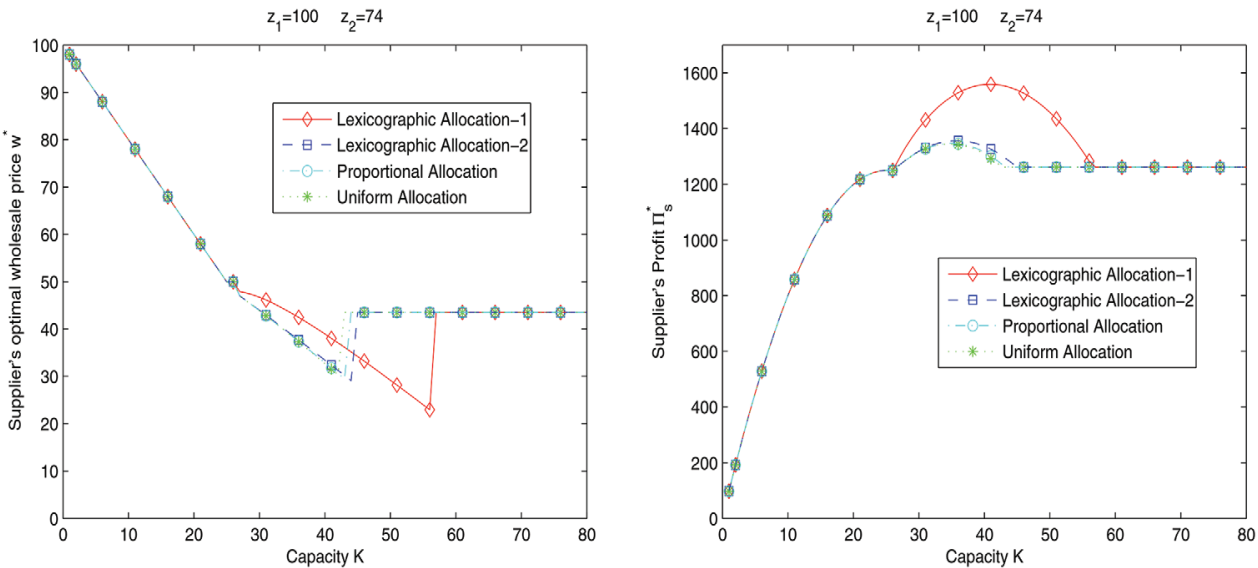

Figure 6. Comparison of Supplier's Pricing Decisions with Asymmetric Retailers. [Color figure can be viewed at wileyonlinelibrary.com]
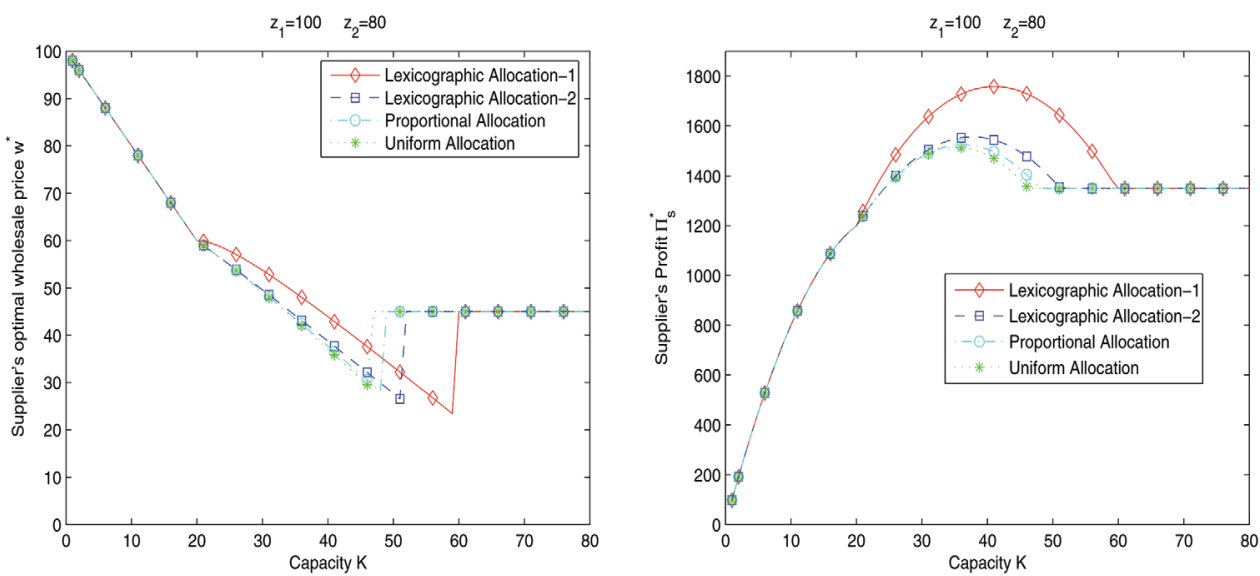

Figure 7. Comparison of Supplier's Pricing Decisions with Asymmetric Retailers. [Color figure can be viewed at wileyonlinelibrary.com] 

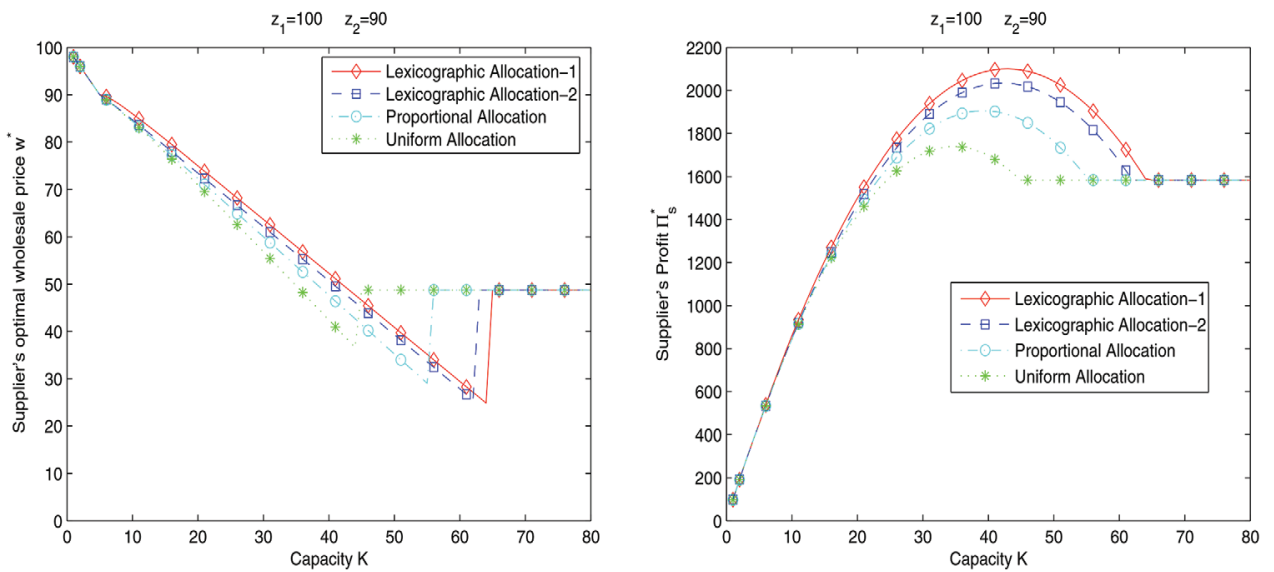

Figure 8. Comparison of Supplier's Pricing Decisions with Asymmetric Retailers. [Color figure can be viewed at wileyonlinelibrary.com]

and $f\left(z_{i}-w\right)=-\left(z_{i}-w\right)^{3}+\left(z_{i}-w\right)^{3}-q_{i}^{* 2}\left(z_{i}-w\right)-q_{i}^{* 2} Q_{-i}^{*}<0$. Consequently, there exists a value $K_{p}^{i *}$ that belongs to $\left[Q^{*}, z_{i}-w\right)$ such that $f(K)=0$. Therefore, $K_{p}^{*}=\max \left\{K_{p}^{1 *}, K_{p}^{2 *}, \ldots, K_{p}^{n *}\right\}$.

Next, we compare the values of $K_{u}^{*}, K_{p}^{*}, K_{l^{*}}^{*}$.

(i) We prove that $K_{u}^{*} \leq K_{p}^{*}$. Consider any retailer $i$ whose profits under proportional and uniform allocations are $\left(z_{i}-w-K\right) \hat{q}_{i}^{\max }$ and $\left(z_{i}-w-K\right) \tilde{q}_{i}^{\max }$, respectively. As $\tilde{q}_{i}^{\max }=\frac{1}{\tilde{n}_{i}^{\prime}+1}(K-$ $\left.\sum_{j=\tilde{n}_{i}^{\prime}+1, \ldots, n ; j \neq i} q_{j}^{*}\right)<\frac{K}{2}<\frac{K}{K+Q_{-i}^{*}} K=\hat{q}_{i}^{\max }$, it follows that $\Pi_{i}\left(\tilde{q}_{i}^{\max }, K-\tilde{q}_{i}^{\max }\right) \leq \Pi_{i}\left(\hat{q}_{i}^{\max }, K-\hat{q}_{i}^{\max }\right)$. Recall that $K_{u}^{i *}$ satisfies $\Pi_{i}\left(\tilde{q}_{i}^{\max }, K-\tilde{q}_{i}^{\max }\right) \geq \Pi_{i}\left(q_{i}^{*}, Q_{-i}^{*}\right)$, and thus $K_{u}^{i *}$ also satisfies $\Pi_{i}\left(\hat{q}_{i}^{\max }, K-\hat{q}_{i}^{\max }\right) \geq \Pi_{i}\left(q_{i}^{*}, Q_{-i}^{*}\right)$, for any $i$. As $K_{p}^{i *}$ is defined as upper bound of $K$ that satisfies $\Pi_{i}\left(\hat{q}_{i}^{\max }, K-\hat{q}_{i}^{\max }\right) \geq$ $\Pi_{i}\left(q_{i}^{*}, Q_{-i}^{*}\right)$, we can derive that $K_{u}^{i^{*}} \leq K_{p}^{i *}$. Hence, we have $K_{u}^{*} \leq K_{p}^{*}$.

(ii) We prove that $K_{p}^{*} \leq K_{l^{*}}^{*}$. Note that $\Pi_{i}\left(\hat{q}_{i}^{\max }, K-\hat{q}_{i}^{\max }\right) \geq$ $\Pi_{i}\left(q_{i}^{*}, Q_{-i}^{*}\right)$ is equivalent to $\left(z_{i}-w-K\right) \frac{K^{2}}{K+Q_{-i}^{*}}-q_{i}^{* 2} \geq 0$ with Eq. (8), and $\Pi_{1}\left(\breve{q}_{1}^{\max }, K-\check{q}_{1}^{\max }\right) \geq \Pi_{1}\left(q_{1}^{*}, Q_{-1}^{*}\right)$ is equivalent to $\left(z_{1}-w-K\right) K-q_{1}^{* 2} \geq 0$.

Note that

$$
\left(z_{1}-w-K\right) K-q_{1}^{* 2} \geq\left(z_{i}-w-K\right) \frac{K^{2}}{K+Q_{-i}^{*}}-q_{i}^{* 2},
$$

which is equivalent to

$$
\begin{aligned}
& {\left[z_{1}-w-Q_{-i}^{*}-\left(z_{i}-w\right)\right] K^{2}} \\
& \quad+\left[\left(z_{1}-w\right) Q_{-i}^{*}-q_{1}^{* 2}+q_{i}^{* 2}\right] K-\left(q_{1}^{* 2}-q_{i}^{* 2}\right) Q_{-i}^{*} \geq 0 .
\end{aligned}
$$

Note that we have

$$
\left(Q^{*}-q_{1}^{*}\right) K^{2}-\left[\left(z_{1}-w\right) Q_{-i}^{*}-q_{1}^{* 2}+q_{i}^{* 2}\right] K+\left(q_{1}^{* 2}-q_{i}^{* 2}\right) Q_{-i}^{*} \leq 0 .
$$

Let $g(K)=\left(Q^{*}-q_{1}^{*}\right) K^{2}-\left[\left(z_{1}-w\right) Q_{-i}^{*}-q_{1}^{* 2}+q_{i}^{* 2}\right] K+\left(q_{1}^{* 2}-q_{i}^{* 2}\right) Q_{-i}^{*}$. We can see that $g\left(Q^{*}\right) \leq 0$ and $g\left(z_{n}-w\right) \leq 0$. Because of the convexity of $g(K)$, we have that $\left(z_{1}-w-K\right) K-q_{1}^{* 2} \geq\left(z_{i}-w-K\right) \frac{K^{2}}{K+Q_{-i}^{*}}-q_{i}^{* 2}$ when $K \in\left[Q^{*}, z_{n}-w\right)$, for any $i$. Thus, we conclude that if $K$ satisfies $\Pi_{i}\left(\hat{q}_{i}^{\max }, K-\hat{q}_{i}^{\max }\right) \geq \Pi_{i}\left(q_{i}^{*}, Q_{-i}^{*}\right)$, then we have that $\Pi_{1}\left(\check{q}_{1}^{\max }, K-\right.$ $\left.\breve{q}_{1}^{\max }\right) \geq \Pi_{1}\left(q_{1}^{*}, Q_{-1}^{*}\right)$, that is, $\left\{K \mid \Pi_{i}\left(\hat{q}_{i}^{\max }, K-\hat{q}_{i}^{\max }\right) \geq \Pi_{i}\left(q_{i}^{*}, Q_{-i}^{*}\right)\right\} \subseteq$
$\left\{K \mid \Pi_{1}\left(\check{q}_{1}^{\max }, K-\check{q}_{1}^{\max }\right) \geq \Pi_{1}\left(q_{1}^{*}, Q_{-1}^{*}\right)\right\}$. Consequently, if the value of $K$ induces the total order quantity to be no less than the supplier's available capacity under proportional allocation, then it also induces retailer 1 to order the whole capacity under lexicographic allocation. Hence, we have $K_{p}^{*} \leq K_{l^{*}}^{*}$.

\section{PROOF OF THEOREM 5:}

(i) When $K_{u}^{*}<K \leq K_{p}^{*}$, the capacity level is sufficiently low such that retailer $i$ 's profit is $\left(z_{i}-w-K\right) q_{i}$, and thus each retailer orders $K$. The unique allocation vector is $(K / n, K / n, \ldots, K / n)$ under uniform and proportional mechanisms. Because retailer 1 has the highest priority under lexicographic allocation, the allocation vector under lexicographic allocation is $(K, 0, \ldots, 0)$. In this case, the total retailer profit $\tilde{\Pi}_{r}=\hat{\Pi}_{r}=\Sigma_{i=1}^{n}\left(z_{i}-w-K\right) \frac{K}{n}$ and $\breve{\Pi}_{r}^{*}=\left(z_{1}-w-K\right) K$. It is easy to verify that $\sum_{i=1}^{n}\left(z_{i}-w-K\right) \frac{K}{n} \leq$ $\left(z_{1}-w-K\right) K$ due to $z_{1} \geq \frac{1}{n} \sum_{i=1}^{n} z_{i}$. Together with the supplier profit, we have the results as shown in case (i).

(ii) When $K_{p}^{*}<K \leq K_{l *}^{*}$, the allocation vector under uniform allocation is $\left(q_{1}^{*}, q_{2}^{*}, \ldots, q_{n}^{*}\right)$. In the meanwhile, allocation vectors under proportional and lexicographic allocations are $(K / n, K / n, \ldots, K / n)$ and $(K, 0, \ldots, 0)$, respectively. Thus, the total retailer profit can be expressed as $\tilde{\Pi}_{r}=\Sigma_{i=1}^{n}\left(z_{i}-w-Q^{*}\right) q_{i}^{*}$, $\hat{\Pi}_{r}=\Sigma_{i=1}^{n}\left(z_{i}-w-K\right) \frac{K}{n}$, and $\check{\Pi}_{r}^{*}=\left(z_{1}-w-K\right) K$. Let $g(K)=$ $\Sigma_{i=1}^{n}\left(z_{i}-w-Q^{*}\right) q_{i}^{*}-\Sigma_{i=1}^{n}\left(z_{i}-w-K\right) \frac{K}{n}$. Note that $g(K)$ is a function of $K$ and is minimized at $K^{*}=\sum_{i=1}^{n}\left(z_{i}-w\right) /(2 n)$. We can prove that $K^{*} \leq Q^{*}$ and $g\left(Q^{*}\right)>0$, and it follows that $g(K)$ is increasing in $K \in\left[Q^{*}, \infty\right)$. Thus, we have $\tilde{\Pi}_{r}^{*} \geq \hat{\Pi}_{r}^{*}$. Similar to case (i), we have $\left(z_{1}-w-K\right) K \geq \Sigma_{i=1}^{n}\left(z_{i}-w-K\right) \frac{K}{n}$. As $\check{\Pi}_{r}^{*}-\tilde{\Pi}_{r}^{*}=K^{2}-\frac{K}{n} \Sigma_{i=1}^{n} z_{i}+\Sigma_{i=1}^{n}\left(z_{i}-Q^{*}\right) q_{i}^{*}+w\left(K-Q^{*}\right)$, taking into consideration the difference in the supplier's profits $w Q^{*}-w K$, we obtain the sufficient and necessary condition for the relationships of $\check{\Pi}_{r}^{*}$ and $\tilde{\Pi}_{r}^{*}$ and of $\check{\Pi}_{s c}^{*}$ and $\tilde{\Pi}_{s c}^{*}$ as shown in case (ii) of the theorem.

(iii) When $K_{p}^{*}<K \leq K_{l *}^{*}$, the unique allocation vector from equilibrium orders under uniform and proportional mechanisms is $\left(q_{1}^{*}, q_{2}^{*}, \ldots, q_{n}^{*}\right)$, while under lexicographic mechanism the unique allocation vector from equilibrium orders is $(K, 0, \ldots, 0)$. It is straightforward to verify that uniform and proportional allocations generate the same total retailer profit $\tilde{\Pi}_{r}^{*}=\hat{\Pi}_{r}^{*}$. Note that under uniform and proportional allocations, the supplier sells $Q^{*}$ units of capacity and achieves profit $w Q^{*}$. Consequently, we have 
$\tilde{\Pi}_{s c}^{*}=\hat{\Pi}_{s c}^{*}$. Following this logic, it is straightforward to prove the remaining results in case (iii) of the theorem.

(iv) When $K>K_{l *}^{*}$, the supplier's capacity is sufficiently high, and under all the three mechanisms, each retailer orders her ideal order size and the resulted allocation vector is $\left(q_{1}^{*}, q_{2}^{*}, \ldots, q_{n}^{*}\right)$. Therefore, the retailers and the supply chain obtain the same profit under the three mechanisms, respectively.

\section{Numerical Computation of Thresholds $K^{*}$}

The thresholds $K_{u}^{*}, K_{p}^{*}, K_{l_{t}}^{*}$ under uniform, proportional, and proportional (with priority sequence $l_{t}$, where retailers are indexed as $1,2, \ldots, n$, without loss of generality) can be characterized by equilibrium ordering, as follows. Given any supplier's wholesale price $w$ and supplier's capacity $K \geq Q^{*}$,

(i) under uniform mechanism, the order quantity vector $\left(q_{1}^{*}, q_{2}^{*}, \ldots, q_{n}^{*}\right)$ is in equilibrium only if $\Pi_{i}\left(\tilde{q}_{i}^{\max }, K-\tilde{q}_{i}^{\max }\right) \leq \Pi_{i}\left(q_{i}^{*}, q_{-i}^{*}\right)$ for any $i$, where $\tilde{q}_{i}^{\max }=\frac{1}{\tilde{n}_{i}^{\prime}+1}\left(K-\sum_{j=\tilde{n}_{i}^{\prime}+1, \ldots, n ; j \neq i} q_{j}^{*}\right)$ and $\tilde{n}_{i}^{\prime}$ is defined as the largest integer less than $i$ such that $q_{\tilde{n}_{i}^{\prime}}^{*} \geq$ $\frac{1}{\tilde{n}_{i}^{\prime}+1}\left(K-\sum_{j=\tilde{n}_{i}^{\prime}+1, \ldots, n ; j \neq i} q_{j}^{*}\right)$;

(ii) under proportional mechanism, the order quantity vector $\left(q_{1}^{*}, q_{2}^{*}, \ldots, q_{n}^{*}\right)$ is in equilibrium only if $\Pi_{i}\left(K^{2} /\left(K+q_{-i}^{*}\right), K-\right.$ $\left.K^{2} /\left(K+q_{-i}^{*}\right)\right) \leq \Pi_{i}\left(q_{i}^{*}, q_{-i}^{*}\right)$ for any $i$

(iii) under lexicographic mechanism, the order quantity vector $\left(q_{1}^{*}, q_{2}^{*}, \ldots, q_{n}^{*}\right)$ is in equilibrium only if $\Pi_{i}(K-$ $\left.\underset{j=1, \ldots, i-1}{\Sigma} q_{j}^{*}, \sum_{j=1, \ldots, i-1}^{\Sigma} q_{j}^{*}\right) \leq \Pi_{i}\left(q_{i}^{*}, q_{-i}^{*}\right)$ for any $i$.

The results are intuitive. Under a specific allocation mechanism, when the supplier's wholesale price $w$ and capacity level $K$ are given, retailer $i$ will order the ideal allocation $q_{i}^{*}$ if the profit generating from the inflated allocation (i.e., ordering as much as possible) is less than the profit resulting from the ideal allocation $\left(q_{1}^{*}, q_{2}^{*}, \ldots, q_{n}^{*}\right)$. In other words, if every retailer's profit satisfies this condition, then all retailers will order their ideal order quantities, that is, the Nash equilibrium order vector $\left(q_{1}^{*}, q_{2}^{*}, \ldots, q_{n}^{*}\right)$, and consequently the total allocation is $Q^{*}$. On the other hand, if there exists at least one retailer $i$, whose profit is larger when ordering as much as possible (i.e., $K$ ) so as to receive the maximum possible allocation given that the total allocation is $K$. Accordingly, the total allocation will be equal to the available capacity $K$, and any retailer will order at least $K / n$ for her best interest. Hence, the equilibrium allocation vector is $(K / n, K / n, \ldots, K / n)$.

Under each allocation mechanism, the minimum value of $K$, given that $K \geq Q^{*}$, satisfying the correspondent condition is the threshold $K^{*}$ for the mechanism. Numerically, it is straightforward to use a binary search to locate the threshold $K^{*}$ with specific precision for each mechanism.

\section{ACKNOWLEDGMENTS}

The authors are grateful to the Editor-in-Chief Professor Awi Federgruen, the Associate Editor, and the two anonymous reviewers for their constructive comments, which substantially improved the quality of our work. The first author's research was supported in part by the Natural Science Foundation of China (No. 71571079). The second author's work was supported in part by the Research Center of Enterprise Decision Support, Key Research Institute of Humanities and Social Sciences in Universities of Hubei Province (DSS20170301) and the Doctoral Scientific Research Foundation of Wuhan Textile University (165022).

\section{REFERENCES}

[1] G. Cachon, M. Lariviere, Capacity choice and allocation: Strategic behavior and supply chain performance, Manage Sci 45 (1999), 1091-1108.

[2] G. Cachon, M. Lariviere, An equilibrium analysis of linear, proportional and uniform allocation of scarce capacity, IIE Trans, 31 (1999), 835-849.

[3] G. Cachon, M. Lariviere, Capacity allocation using past sales: When to turn-and-earn, Manage Sci 45 (1999), 685-703.

[4] F. Chen, J. Li, H. Zhang, Managing downstream competition via capacity allocation, Prod Oper Manage, 22 (2013), $426-446$.

[5] Y. Chen, X. Su, X. Zhao, Modeling bounded rationality in capacity allocation games with the quantal response equilibrium, Manage Sci 58 (2012), 1952-1962.

[6] S.-H. Cho, C. Tang, Capacity allocation under retail competition: uniform and competitive allocations, Oper Res 62 (2014), $72-80$.

[7] N.G. Hall, Z. Liu, "Capacity allocation in supply chain scheduling," in: J.J. Cochran (editor), Wiley encyclopedia of operations research and management science, Wiley, New York City, New York, 2010, pp. 576-584.

[8] A.V. Iyer, Z.W. Deshpande, A postponement model for demand management. Manage Sci 49 (2003), 983-1002.

[9] H.L. Lee, V. Padmanabhan, S.J. Whang, Information distortion in a supply chain: the bullwhip effect, Manage Sci 43 (1997), $546-558$.

[10] Z. Liu, Equilibrium analysis of capacity allocation with demand competition. Nav Res Logist, 59 (2012), 254-265.

[11] Y. Sprumont, The division problem with single-peaked preferences: A characterization of the uniform allocation rule. Econometrica 59 (1991), 509-519.

[12] Z. Yang, X. Hu, H. Gurnani, H. Guan, Selling to a competing buyer with limited supplier capacity, Manage Sci (in press). 